\title{
1 An electrophysiological and behavioral model of Paramecium, the 2 "swimming neuron"
}

3 Irene Elices ${ }^{1}$, Anirudh Kulkarni1 ${ }^{1,2}$, Nicolas Escoubet ${ }^{3}$, Léa-Laetitia Pontani ${ }^{3}$, Alexis Michel Prevost 3 ,

4 Romain Brette ${ }^{1}$

51 Sorbonne Université, INSERM, CNRS, Institut de la Vision, 17 rue Moreau, F-75012 Paris,

6 France

$7 \quad 2$ Department of Bioengineering and Centre for Neurotechnology, Imperial College London, South

8 Kensington Campus, SW7 2AZ London, United Kingdom

93 Sorbonne Université, CNRS, Institut de Biologie Paris-Seine (IBPS), Laboratoire Jean Perrin

10 (LJP), F-75005, Paris

Corresponding author: Romain Brette, romain.brette@inserm.fr

\section{Abstract}

Paramecium is a large unicellular organism that swims in fresh water using cilia. When stimulated by various means (mechanically, chemically, optically, thermally), it often swims backward then turns and swims forward again in a new direction: this is called the avoiding reaction. This reaction is triggered by a calcium-based action potential. For this reason, several authors have called Paramecium the "swimming neuron". Here we present an empirically constrained model of its action potential based on electrophysiology experiments on live immobilized paramecia, together with simultaneous measurement of ciliary beating using particle image velocimetry. Using these measurements and additional behavioral measurements of free swimming, we extend the electrophysiological model by coupling calcium concentration to kinematic parameters, turning it into a swimming model. In this way, we obtain a model of autonomously behaving Paramecium. Finally, we demonstrate how the modeled organism interacts with an environment, can follow gradients and display collective behavior. This work provides a modeling basis for investigating the physiological basis of autonomous behavior of Paramecium in ecological environments. 


\section{Introduction}

Behavior depends on a complex interaction between a variety of physiological processes, the body and the environment. This complexity makes it challenging to develop integrative models relating the different components. Thus, a strategy is to study model organisms that are experimentally accessible and structurally simpler than vertebrates. This strategy has been applied in particular to C. Elegans, with its 302 neurons and a known connectome (Cohen and Denham, 2019; Cohen and Sanders, 2014; Schafer, 2018). However, electrophysiology is difficult owing to the small size of its neurons (about 3 $\mu \mathrm{m})$, and developing empirically valid neuromechanical models of $C$. Elegans remains challenging. More recently, other model organisms have been introduced: Hydra, with a few thousand neurons and the advantage of being transparent (Dupre and Yuste, 2017; Wang et al., 2020), and jellyfish Aurelia aurita (Pallasdies et al., 2019).

Here we propose to develop an integrative model of Paramecium tetraurelia (Brette, 2021), which is structurally much simpler than the abovementioned model organisms, since it is a unicellular organism, while being large enough to perform intracellular electrophysiology (about $120 \mu \mathrm{m}$ long (Nagel and Machemer, 2000)). Paramecium is a common ciliate, which swims in fresh water by beating its $\sim 4000$ cilia (Aubusson-Fleury et al., 2015; Iftode et al., 1989; Nagel and Machemer, 2000), and feeds on smaller microorganisms (bacteria, algae, yeast). It uses chemical signals to find food, avoids obstacles thanks to mechanosensitivity, displays collective behavior, adapts to changing environmental conditions and can even learn to respond to new stimuli (Hennessey et al., 1979).

More than a century ago, Jennings described the basis of its behavior as "trial-and-error" (Jennings, 1906). Paramecium normally swims in a helicoidal fashion at about 500-1000 $\mu \mathrm{m} / \mathrm{s}$, but when it encounters something undesirable (obstacle, hot region, noxious substance), it produces an avoiding reaction (Fig. 1A): it briefly swims backward, then turns and swims forward in a new direction. The avoiding reaction is triggered by a calcium-based graded action potential, which can be observed in an immobilized cell in response to a current pulse (Fig. 1B). The calcium current is produced by L-type calcium channels located in the cilia (Eckert, 1972), related to the Cav1 family found in neurons, heart and muscles of mammals (Lodh et al., 2016). Genes for many ionic channels have been found in the fully sequenced genome (Martinac et al., 2008), and a number have been electrophysiologically identified (Eckert and Brehm, 1979). Many signaling pathways of neurons have been found in 58 Paramecium (Plattner and Verkhratsky, 2018). For this reason, Paramecium has been called a 59 "swimming neuron" (Kung and Saimi, 1985) and there is a vast amount of information about its electrophysiology, from studies done mainly in the 1960-80s (Eckert, 1972; Eckert and Brehm, 1979). However, there is no empirically based model of its action potential. it a model of autonomously behaving organism. It is based on electrophysiological experiments on immobilized paramecia with simultaneous imaging of fluid motion induced by cilia beating, and behavioral measurements. We then demonstrate how the modeled organism interacts with the environment, can display chemotaxis and collective behavior. 

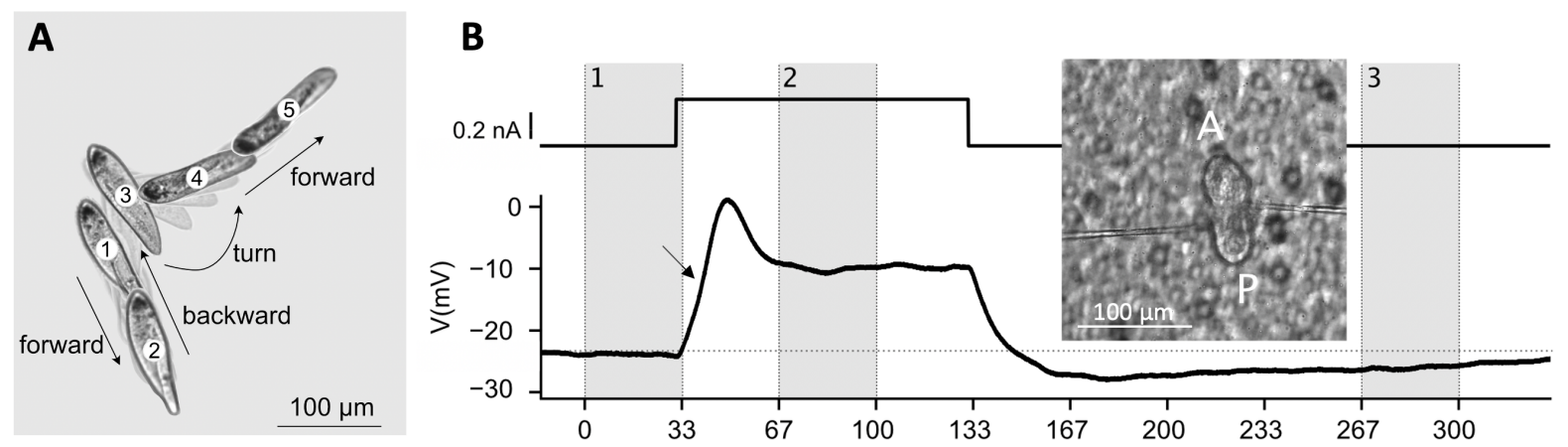

C1 $\mathrm{t}=0-33 \mathrm{~ms}$

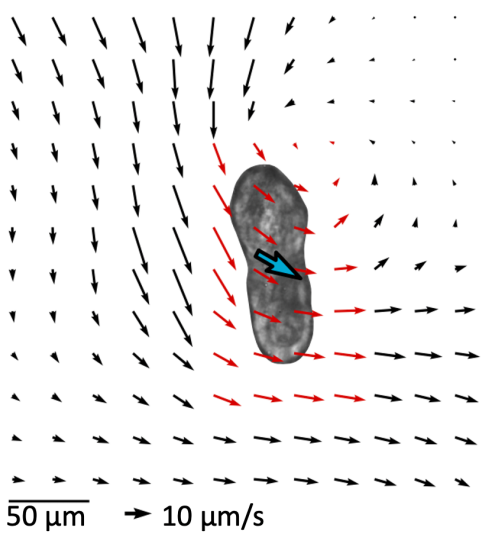

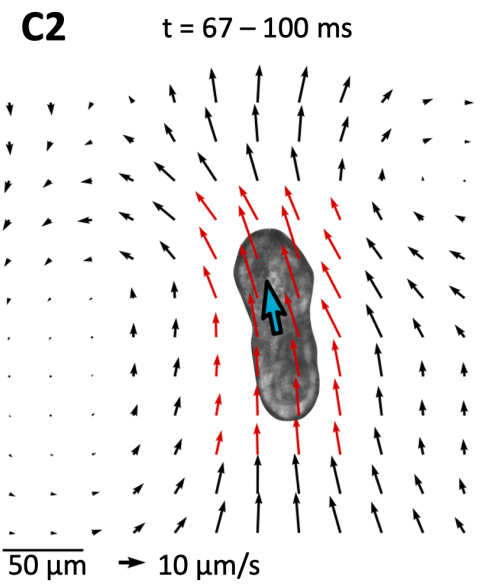

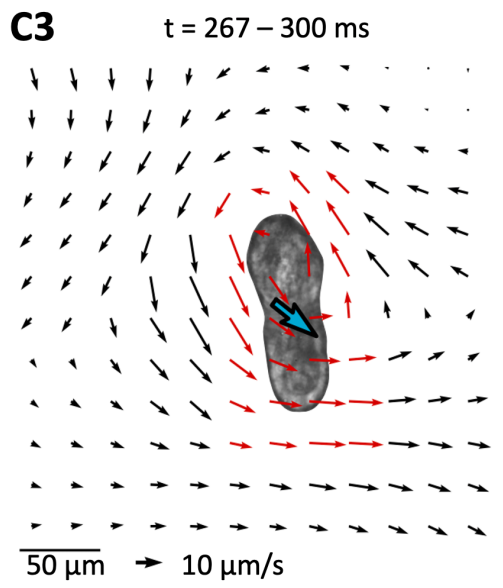

Figure 1. The avoiding reaction of Paramecium. A, Typical spontaneous avoiding reaction: the ciliate swims backward, then turns and eventually resumes forward swimming, while spinning around its main axis during the entire movement. Images are separated by $150 \mathrm{~ms}$, with intermediate shaded frames every $37 \mathrm{~ms}$. The cell was placed in $20 \mathrm{mM} \mathrm{NaCl}$ and $0.3 \mathrm{mM} \mathrm{CaCl} 2$ to induce spontaneous avoiding reactions (Kung, 1971). B, Intracellular recording of a voltage response (bottom right) to a square current pulse of amplitude $300 \mathrm{pA}$ (top right) in an immobilized cell (left; A: anterior end; P: posterior end), showing a small action potential (in the standard extracellular solution, see Methods). The arrow points at a small upward inflexion due to the calcium current. Inset: Paramecium immobilized on a filter (background) with two electrodes. C, Velocity field of the fluid on a plane $\sim 30 \mu \mathrm{m}$ above the cell, calculated over the three shaded intervals shown in B. The blue arrow indicates mean velocity in the whole field, represented twice larger for clarity. C1, The fluid moves backward, which would make the cell swim forward. C2, The fluid moves forward. C3, The flow direction reverts on the posterior end, but not on the anterior right end, resulting in a swirling pattern.

Results

\section{A brief overview of Paramecium's action potential}

To perform intracellular electrophysiology (see Methods, Electrophysiology), it is necessary to first immobilize the cell. To this end, we use a device we previously developed (Kulkarni et al., 2020), which uses a transparent filter with holes smaller than the cell and a peristaltic pump. The pump draws the extracellular solution ( $4 \mathrm{mM} \mathrm{KCl}$ and $1 \mathrm{mM} \mathrm{CaCl}_{2}$ ) from an outlet below the filter, immobilizing the cells against the filter (Fig. 1B, inset). Two high-resistance electrodes are then inserted into the cell and the pump is stopped. The cell is then held by the electrodes. One electrode is used to inject current, while the other is used to measure the membrane potential. Paramecium is a large cell (about $120 \mu \mathrm{m}$ long and $35 \mu \mathrm{m}$ wide for P. tetraurelia), which makes it isopotential (Eckert and Naitoh, 1970; Satow and Kung, 1979). 
Depolarization opens voltage-gated calcium channels located in the $\sim 4000$ cilia (Aubusson-Fleury et al., 2015; Iftode et al., 1989; Nagel and Machemer, 2000), similar to the L-type Cav1.2 family in mammals (Lodh et al., 2016). This can be noticed on Fig. 1B as a small upward deflection before the peak of the membrane potential (arrow). This calcium current, denoted as $I_{\mathrm{Ca}}$, activates rapidly (a few milliseconds). Calcium entry then makes cilia reorient, which makes them beat forward.

To observe the beating direction, we use $1 \mu \mathrm{m}$ tracer particles and estimate their velocity with particle image velocimetry (PIV, see Methods), on a plane about $30 \mu \mathrm{m}$ above the cell (Fig. 1C1-C3, note that fluid velocity is much smaller than swimming velocity as it is measured above the cell (Drescher et al., 2010)). Before the stimulation, the cilia beat backward to the right (Fig. 1C1), which tends to make the cell move forward, with a spiraling movement over to the left. When calcium enters the cilia, cilia reorient and beat backward, slightly to the left (Fig. 1C2), which makes the cell move backward. The calcium channels inactivate rapidly (a few milliseconds) through calcium-mediated inactivation: intraciliary calcium binds to calmodulin, which then inactivates the channels (Brehm et al., 1980; Eckert and Brehm, 1979; Eckert and Chad, 1984). Calcium is then expelled by diffusion, buffering and pumps. After calcium has entered the cilia, voltage-gated $\mathrm{K}^{+}$channels located in the basal membrane rapidly open, producing a delayed rectifier current $\mathrm{I}_{\mathrm{Kd}}$ that damps the membrane potential (Fig. 1B,

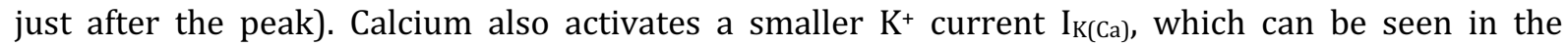
prolonged hyperpolarization after the stimulation.

111 After the stimulation, when calcium concentration has decreased below $\sim 1 \mu \mathrm{M}$ (Naitoh and Kaneko, 1972), cilia revert to backward beating (Fig. 1C3). We notice a swirling pattern on Fig. 1C3, which can be attributed to an asynchronous reversal of different groups of cilia. We will show how this relates to the change in swimming direction seen on Fig. $1 \mathrm{~A}$.

We used current-clamp recordings and PIV measurements of fluid motion to build a model of the action potential together with electromotor coupling. We chose to use current-clamp rather than voltage-clamp recordings because good control is difficult to achieve with high resistance electrodes and several important processes are calcium-gated rather than voltage-gated, making voltage-clamp less relevant.

\section{Passive properties}

We start by estimating the passive properties (resistance, capacitance, reversal potentials) with model fitting techniques (see Methods, Electrophysiological modeling and Model optimization), and we compare with previous measurements in the literature. To this end, we use voltage responses to hyperpolarizing current pulses (duration $100 \mathrm{~ms}$, amplitude 0 to $-4 \mathrm{nA}$ in $300 \mathrm{pA}$ increments; Fig. 2A, top). Such stimuli are known to trigger different voltage-gated currents: a small inactivating calcium current (Nakaoka and Iwatsuki, 1992; Preston et al., 1992a, 1992b), and a strong inward rectifier $\mathrm{K}^{+}$

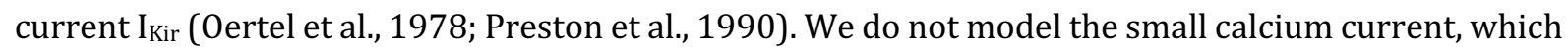
is related to the escape reaction, an increase in swimming speed triggered by hyperpolarizing stimuli. We only model the inward rectifier current, which will allow us to estimate $E_{K}$, the reversal potential of $\mathrm{K}^{+}$.

When the pulse intensity is strong, an inward current can be seen to activate after $\sim 15 \mathrm{~ms}$. The inward rectifier current has the property to activate mainly below $\mathrm{E}_{K}(\mathrm{Lu}, 2004)$. This can be seen by removing $\mathrm{K}^{+}$from the extracellular solution (making $E_{K}=-\infty$ ). With $4 \mathrm{mM}$ extracellular $\mathrm{KCl}$, hyperpolarization below about $-60 \mathrm{mV}$ activates a strong inward current, which is largely suppressed in $0 \mathrm{mM} \mathrm{KCl} \mathrm{(Fig.}$ 2B). After the pulse, the $\mathrm{K}^{+}$current switches from inward to outward as it passes $\mathrm{E}_{\mathrm{K}}$. This results in a 
A
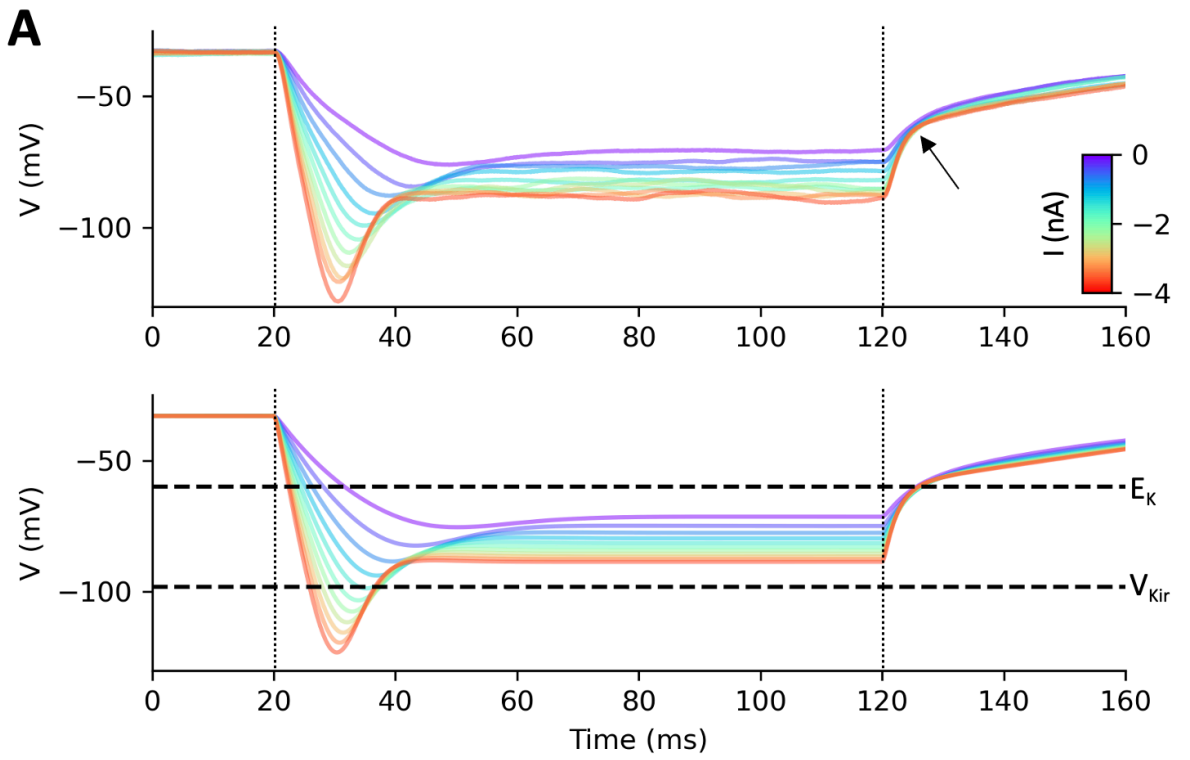

D

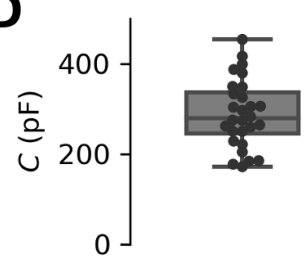

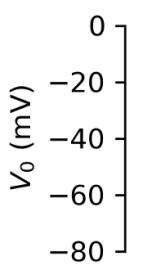
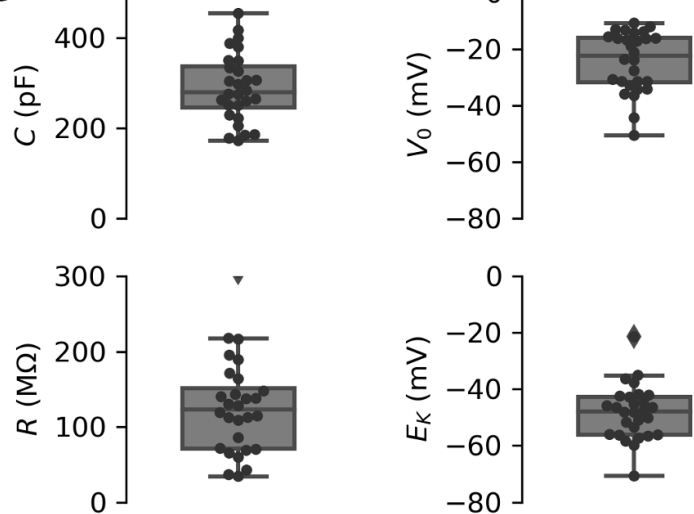

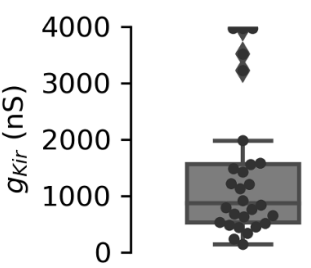

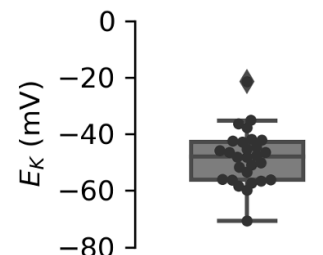

Passive

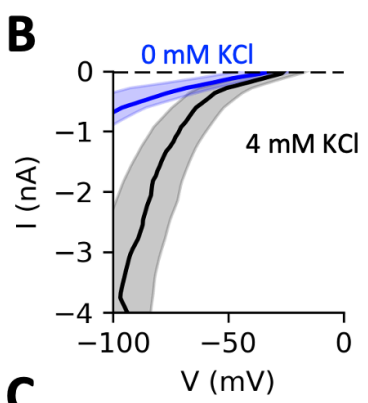

C
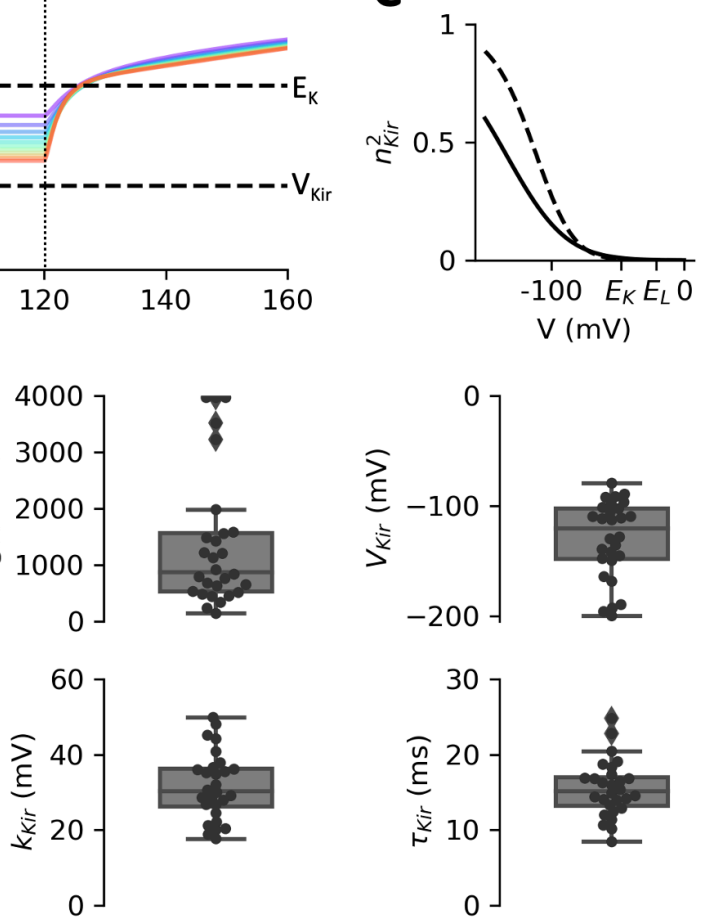
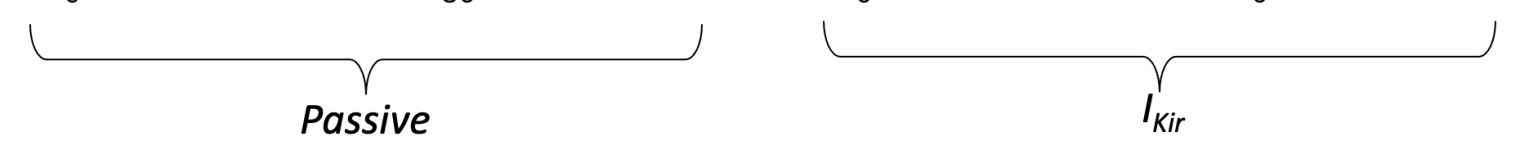

Figure 2. Passive properties and inward rectifier current. A, Top: voltage responses of one cell to negative current pulses ( $I=0$ to $-4 \mathrm{nA}$ in $300 \mathrm{pA}$ increments; dashed lines: start and end of pulses), in the standard extracellular solution ( $4 \mathrm{mM} \mathrm{KCl}$ and $1 \mathrm{mM} \mathrm{CaCl} 2$ ). The arrow points at an inflexion due to the inward rectifier current $I_{K i r}$. Bottom: model responses fitted to the data, showing the inferred reversal potential of $K^{+}\left(E_{K}\right)$ and the half-activation voltage $V_{\text {Kir }}$ of the inward rectifier current. B, Current-voltage relationship over all cells (mean \pm standard deviation, measured at pulse end) in $4 \mathrm{mM} \mathrm{KCl}$ (grey) and $0 \mathrm{mM} \mathrm{KCl}$ (blue). Removing $\mathrm{K}^{+}$ from the extracellular solution largely suppresses the inward current. C, Activation curve of the inward rectifier current in the fitted models. The current activates below $E_{K}\left(E_{L}\right.$ is leak reversal potential). The solid curve is the activation function with median parameters, the dashed curve is the activation function of the cell shown in $A$. $D$, Fitted parameters over $n=28$ cells, grouped in passive parameters and inward rectifier parameters.

To this end, we fit a biophysical model consisting of a linear leak current and an inward rectifier current $\mathrm{I}_{\mathrm{Kir}}$ (Fig. 2A, bottom) (see Methods, Electrophysiological modeling) using the Brian 2 simulator (Stimberg et al., 2019) with the model fitting toolbox (Teska et al., 2020), which applies differential evolution and gradient descent for least square estimation of model parameters. 
155 We modeled the inward rectifier current as a non-inactivating current with Boltzmann activation, two 156 gates and a fixed time constant: $I_{K i r}=g_{K i r} n_{K i r}^{2}\left(E_{K}-V\right)$ (equations (4-5)). Figure 2C shows the 157 activation curve $n_{\text {Kir }}^{2}(V)$ of the cell shown in Fig. 2A (dashed) and the curve with median parameters 158 (solid), which confirms that the current activates essentially below $\mathrm{E}_{\mathrm{K}}$.

159 We find that the resting potential $V_{0}$ is $-24.5 \mathrm{mV} \pm 10.6 \mathrm{mV}(\mathrm{n}=28$; median $-22.5 \mathrm{mV}$; Fig. 2D). Oertel 160 et al. (1977) previously reported about $-23 \mathrm{mV}$ with a slightly different extracellular solution. 161 Capacitance $C$ is $289 \pm 75 \mathrm{pF}$. By comparison, $P$. caudatum, which is larger, has a capacitance of about $162700 \mathrm{pF}$. Since P. caudatum is $200 \mu \mathrm{m}$ long and $46 \mu \mathrm{m}$ wide (Machemer and Ogura, 1979) and $P$. 163 tetraurelia is $115 \mu \mathrm{m}$ long and $34 \mu \mathrm{m}$ wide (Nagel and Machemer, 2000), a simple scaling would predict 164 a capacitance $(200 \times 46) /(115 \times 34) \approx 2.35$ times smaller for $P$. tetraurelia, i.e. about $300 \mathrm{pF}$, which is 165 consistent with our estimates.

166 Resistance at rest $R$ (including the contribution from $I_{K i r}$ ) is $126 \pm 62 \mathrm{M} \Omega$. Finally, we find $\mathrm{E}_{\mathrm{K}}=-48 \pm$ $16710 \mathrm{mV}$, corresponding to an intracellular $\mathrm{K}^{+}$concentration $\left[\mathrm{K}^{+}\right]_{\mathrm{i}}=29 \pm 11 \mathrm{mM}$. This is consistent with 168 estimates in the literature obtained with various methods, varying between 18 and $34 \mathrm{mM}$ (Hansma, 169 1974; Oertel et al., 1978; Ogura and Machemer, 1980; Oka et al., 1986).

170 We briefly describe the parameter estimation results for $I_{K i r}$, even though these will not be further 171 used, as only $E_{K}$ plays a role in depolarized responses. The estimates for total conductance $\mathrm{g}_{\mathrm{Kir}}$ and half172 activation voltage $V_{\text {Kir }}$ are variable across cells, presumably because these parameters are not well 173 constrained by the data ( $g_{K i r}$ and $V_{K i r}$ cannot be estimated independently in the voltage region where 174 channels are mostly closed). Nonetheless, the results confirm that $I_{K i r}$ activates essentially below $E_{K}$. 175 Activation slope ( $\left.k_{K i r}=32 \pm 9 \mathrm{mV}\right)$ and time constant $\left(\tau_{\text {Kir }}=16 \pm 4 \mathrm{~ms}\right)$ are better constrained. With 176 the estimated parameters, the inward rectifier current contributes about $14 \%$ of the resting 177 conductance (median; $16 \pm 14 \%$ ). 


\section{A model of the deciliated cell}
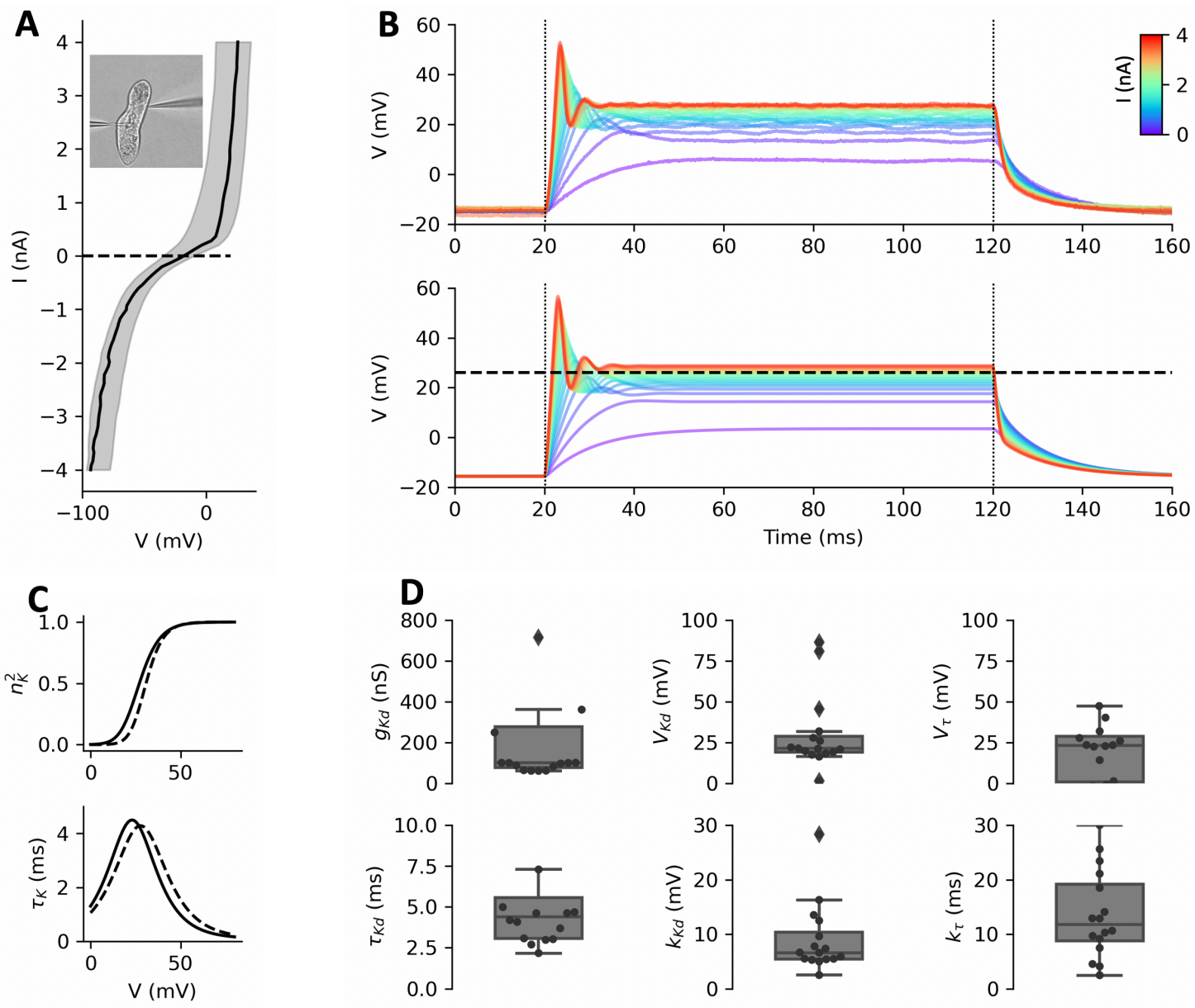

Figure 3. The delayed rectifier current measured in deciliated cells. A, Current-voltage relationship in deciliated cells, showing a strong delayed rectifier current for depolarized voltages. B, Top: voltage responses of one cell to positive current pulses ( $I=0$ to $4 \mathrm{nA}$ in $300 \mathrm{pA}$ increments). Bottom: responses of the two-gate Boltzmann model fitted to the data, showing the inferred half-activation voltage of the delayed rectifier current (dashed). C, Activation and time constant of the delayed rectifier current as a function of voltage in fitted models, with median parameters (solid) and for the cell shown in B (dashed). D, Statistics of fitted parameters $(n=16)$.

Next, we analyzed the delayed rectifier current $\mathrm{I}_{\mathrm{Kd}}$ responsible for repolarization. The ciliary calcium channel can be pharmacologically blocked with W-7, but this drug is toxic to Paramecium (Hennessey and Kung, 1984). Instead, we isolated the delayed rectifier current mechanically by removing the cilia with ethanol (Machemer and Ogura, 1979; Ogura, 1981) (see Methods, Deciliation). This procedure does not kill the cell, and cilia grow back after a few hours. It removes the voltage-gated calcium channels, which are located in the cilia (Lodh et al., 2016; Machemer and Ogura, 1979), and thereby also removes the calcium-activated $\mathrm{K}^{+}$current $\mathrm{I}_{\mathrm{K}(\mathrm{Ca}) \text {. }} \mathrm{In}$ addition, it is no longer necessary to use the immobilization device (Fig. 3A, inset). As can be seen on Fig. 3A, the membrane still produces a strong outward delayed rectifier current upon depolarization, and a strong inward current upon hyperpolarization.

We fitted a Boltzmann model of the delayed rectifier current (see Methods, Electrophysiological modeling, equations (6-7) and (12-13)), $I_{K d}=g_{K d} n^{2}\left(E_{K}-V\right)$, to responses to $100 \mathrm{~ms}$ depolarizing current pulses ( 0 to $4 \mathrm{nA}$ in $300 \mathrm{pA}$ increments). This model turned out to fit the data as well as a 
Hodgkin-Huxley model, but with fewer parameters (see Methods, Model optimization). Figure 3B (bottom) shows responses of this model, and Figure 3C shows the activation curve and voltagedependent time constant with median parameters and those for the cell shown in Fig. 3B, with detailed statistics in Figure 3D. The delayed rectifier current activates at a median value of $V_{K d} \approx 21 \mathrm{mV}$ (30 $\pm 23 \mathrm{mV}$ ) with a slope $k_{K d} \approx 7 \mathrm{mV}(9 \pm 6 \mathrm{mV})$. The time constant peaks at $\sim 4.1 \mathrm{~ms}(4 \pm 1.3 \mathrm{~ms})$ at a voltage $V_{\tau} \approx 23 \mathrm{mV}(26 \pm 48 \mathrm{mV})$, with a slope $k_{\tau} \approx 12 \mathrm{mV}(14 \pm 8 \mathrm{mV})$.

Based on these results, we further simplified the model by enforcing $V_{K d}=V_{\tau}$ and $k_{\tau}=2 k_{K d}$. This simplification slightly increases the fit error (1.82 vs. $1.8 \mathrm{mV} ; \mathrm{p}=0.009$, two-tailed Wilcoxon test), but reduces the number of parameters. We used this simplified model in the full model of ciliated cells (leaving its parameters unconstrained).

\section{The action potential}

214 We now build a model of the action potential of ciliated cells, coupled with cilia reversal (Fig. 4A). Cilia 215 revert with very small depolarizations, of just a few $\mathrm{mV}$ (Machemer, 1974). For this reason, we used 216 two sets of pulses, large pulses from 0 to $5 \mathrm{nA}$ in $300 \mathrm{pA}$ increments, and small pulses from -100 to 500 217 pA in 25 pA increments (Fig. 4A, bottom). Tip potentials could fluctuate between these two sets, 218 therefore we aligned the traces to the median resting potential of $-22 \mathrm{mV}$, and we fixed $\mathrm{E}_{\mathrm{K}}$ at its median value of $-48 \mathrm{mV}$. Simultaneously, we seeded the extracellular medium with $1 \mu \mathrm{m}$ tracer particles and imaged their motion at a frame rate of $30 \mathrm{~Hz}$. Particle image velocimetry was then used to calculate the fluid velocity field, giving an indication of the direction of ciliary beating, as illustrated in Fig. 1C (See Methods, Particle image velocimetry). Figure 4B shows the cosine of the mean angle $\alpha$ of the velocity field during stimulation, relative to the cell's anteroposterior axis: 1 means that particles flow towards the posterior end, i.e., the cell is trying to swim forward; -1 means that the cell is trying to swim backward. Thus, cilia revert for a duration longer than the pulse (120-200 ms in this cell), graded with pulse intensity. 

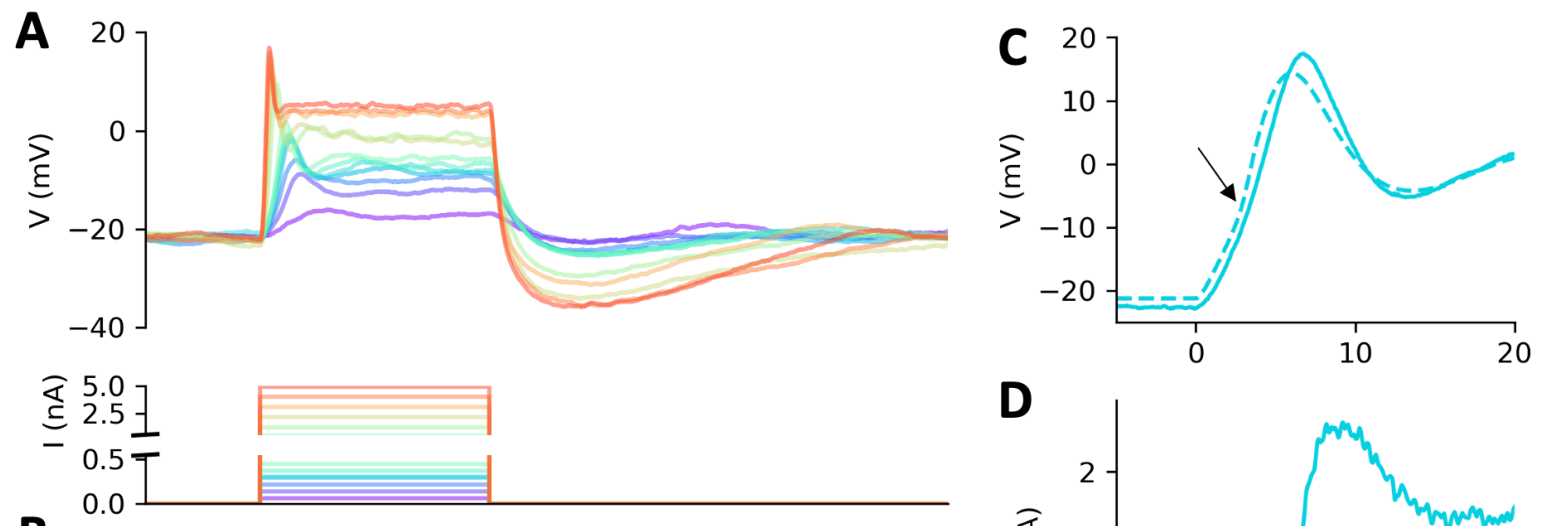

B
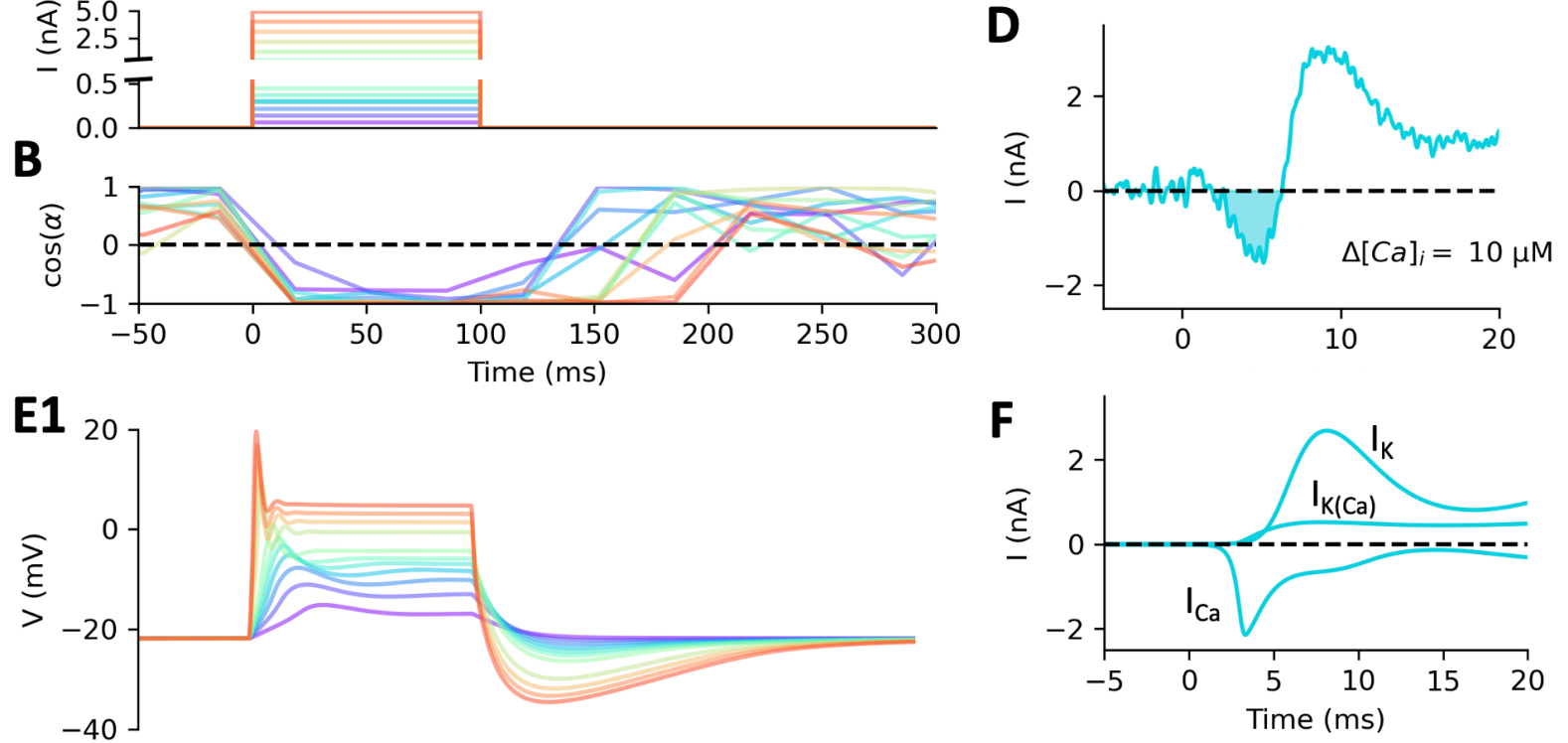

\section{E2}
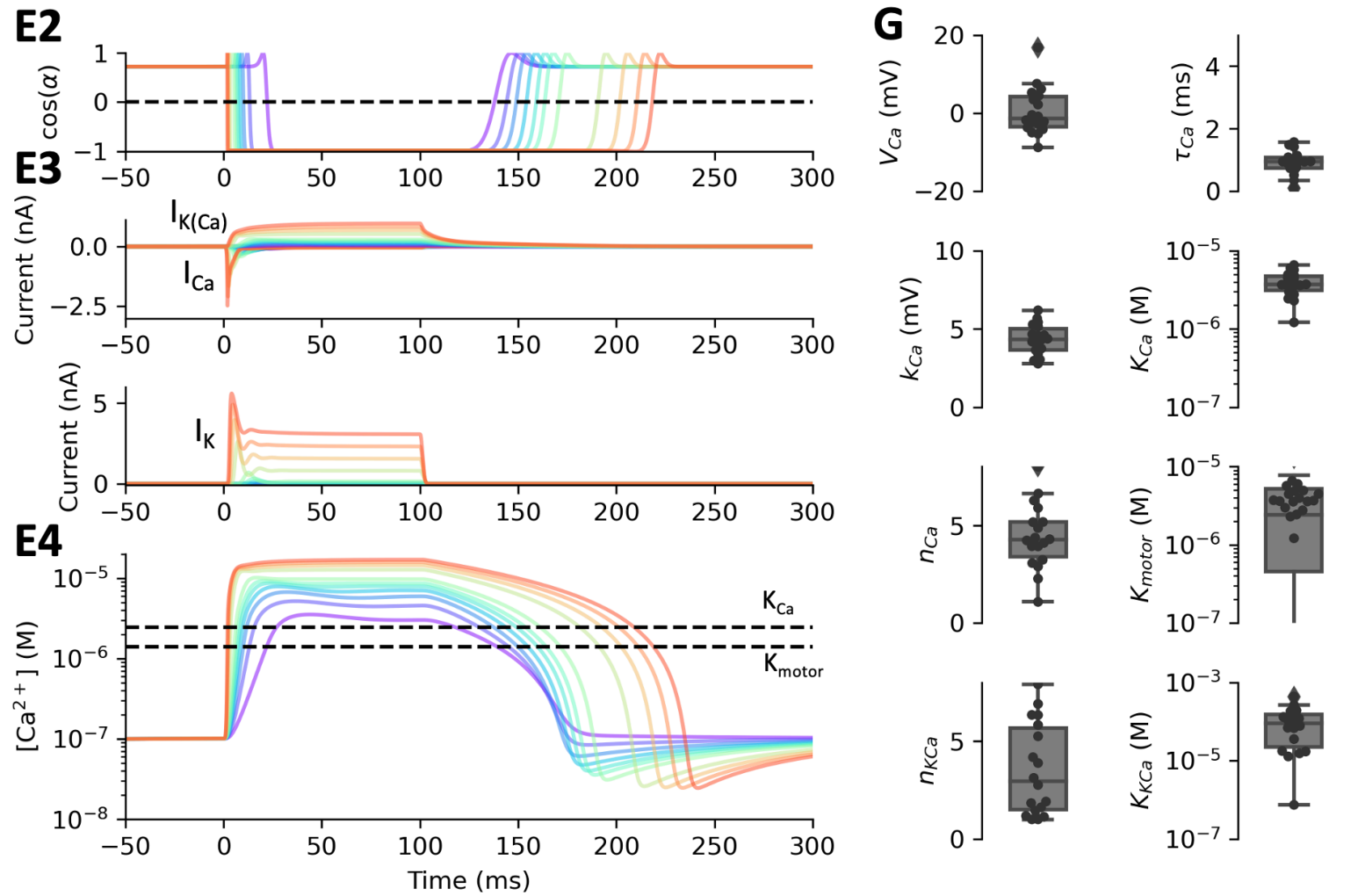

228 Figure 4. Fitting the action potential of Paramecium. A, Voltage responses of a cell (top) to two sets of current pulses (bottom), from 0 to $5 \mathrm{nA}$ (in $300 \mathrm{pA}$ increments) and from -100 to $300 \mathrm{pA}$ (in $25 \mathrm{pA}$ increments). $B$, Ciliary response to the same currents, measured as the cosine of the mean angle of the velocity field, relative to the anteroposterior axis. $C$, Close up of an action potential triggered by a $1.5 \mathrm{nA}$ current pulse, with the model fit (dashed). The arrow points at an upward deflection due to the calcium current. D, Ionic current calculated 
by subtracting the estimated leak current from the capacitive current. The inward current (shaded) corresponds to the calcium current. Integrating this current yields a calcium entry corresponding to a $10 \mu \mathrm{M}$ increase in intraciliary calcium concentration. E, Responses of the fitted model. E1, Voltage responses. E2, Ciliary responses. E3, Voltage-gated calcium current ICa (top, negative traces), delayed rectifier $K^{+}$current $I_{K d}$ (bottom) and calcium-activated $K^{+}$current $I_{K(C a)}$ (top, positive traces) in the fitted model. E4, Intraciliary calcium concentration in the fitted model. The dashed lines show the ciliary reversal threshold and the halfinactivation concentration. $F$, Ionic currents inferred by the model for the action potential shown in $C . G$, Statistics of fitted parameters $(n=18)$.

In the electrophysiological responses, we notice several differences with deciliated cells. First, an upward deflection is apparent after stimulation, as illustrated in Fig. 4C (arrow). This deflection is due to an inward current, the $\mathrm{Ca}^{2+}$ current. This current can be estimated by subtracting the estimated leak current from the capacitive current (passive properties estimated by model fitting, see below). With a pulse of intensity I = $1.5 \mathrm{nA}$, we find that the inward part of that current peaks at about $-2 \mathrm{nA}$ (Fig. 4D). This is an underestimation since part of the inward current may be masked by the $\mathrm{K}^{+}$current, but it is comparable to previous estimations in voltage-clamp (Oertel et al., 1977). This current is known to activate and inactivate quickly, within a few ms (Brehm et al., 1980; Oertel et al., 1977), as can be seen on Fig. 4D. By integrating this current, we calculate that it should lead to an increase in intraciliary calcium concentration of about $\int I / 2 v F=10 \mu \mathrm{M}$ (a lower estimate, because of masking by $\mathrm{K}^{+}$currents), where $v \approx 1700 \mathrm{\mu m}^{3}$ is the estimated ciliary volume (see Methods, Electrophysiological modeling) and $F$ is the Faraday constant. This is well above the threshold for ciliary reversal, which has been estimated at about $1 \mu \mathrm{M}$ by exposing Triton-extracted cells to variable concentrations of calcium (Naitoh and Kaneko, 1972).

In the electrophysiological response (Fig. 4A), we also observe small oscillations, due to the interplay between $\mathrm{Ca}^{2+}$ and $\mathrm{K}^{+}$currents, and a pronounced hyperpolarization after the pulse. This hyperpolarization is due to a calcium-activated $\mathrm{K}^{+}$current $\mathrm{I}_{\mathrm{K}(\mathrm{Ca})}$. This current has been previously characterized electrophysiologically (Saimi et al., 1983; Satow and Kung, 1980), as well as genetically and with immunochemistry (Valentine et al., 2012; Yano et al., 2013).

Thus, we included the following currents in our model: a leak current $\mathrm{I}_{\mathrm{L}}$, a voltage-gated calcium current $\mathrm{I}_{\mathrm{Ca}}$, with calcium-mediated inactivation, a delayed rectifier $\mathrm{K}^{+}$current $\mathrm{I}_{\mathrm{Kd}}$, and a calciumactivated $\mathrm{K}^{+}$current $\mathrm{I}_{\mathrm{K}(\mathrm{Ca})}$ (see Methods, Electrophysiological modeling). The calcium current $\mathrm{I}_{\mathrm{Ca}}$ is produced by ciliary channels similar to L-type Cav1.2 channels (Lodh et al., 2016). We modeled it similarly to (Eckert and Chad, 1984; Standen and Stanfield, 1982), but we allow for several inactivation binding sites (equations (14-16)). In addition, the current uses the Goldman-Hodgkin-Katz equation, which is more appropriate than the linear driving force $\left(\mathrm{E}_{\mathrm{Ca}}-\mathrm{V}\right)$ when intra- and extracellular concentrations are very different (Hille, 2001). The calcium-activated $\mathrm{K}^{+}$current $\mathrm{I}_{\mathrm{K}(\mathrm{Ca})}$ is simply modelled with a conductance increasing as a Hill function of calcium concentration (equations (17269 18)).

In addition, the model must include calcium dynamics (equation (19)). The decay of calcium concentration after the action potential may be due to a combination of processes, including diffusion towards the base, buffering (in particular by centrin), and pumps. We model this combination by a simple linear model of the calcium flux. However, this is not sufficient because there is a large calcium flux at rest through the voltage-gated calcium channels, which must also be expelled or buffered. This can be shown by a simple calculation: if the calcium current is $\sim 2 \mathrm{nA}$ when the membrane is depolarized by $\sim 20 \mathrm{mV}$, then at rest the current should be about $e^{-20 \mathrm{mV} / k} \times 2 \mathrm{nA}$ where $k$ is the activation slope factor; with $k=4 \mathrm{mV}$, we get $13 \mathrm{pA}$, corresponding to an influx $J_{\text {rest }}=\frac{13 \mathrm{pA}}{2 v F} \approx 40 \mu \mathrm{M} / \mathrm{s}$. Thus, we postulate that the resting calcium concentration, about $0.1 \mu \mathrm{M}$ (Iwadate, 2003; Klauke and 
Plattner, 1997), is maintained by a pump operating near that concentration, which is consistent with properties of plasma membrane calcium pumps (PMCA), also present in the cilia (Yano et al., 2015, 2013). We model this pump with Michaelis-Menten kinetics.

282 Finally, we couple calcium concentration with ciliary beating angle by a Hill function (equation (20)).

We then fitted this complete model simultaneously to electrophysiological and motor responses to 100 ms current pulses $(n=18)$, while ensuring that the resting calcium concentration was $0.1 \mu \mathrm{M}$ (Fig. 4E). Thus, calcium concentration is not directly measured, but indirectly constrained by several processes:

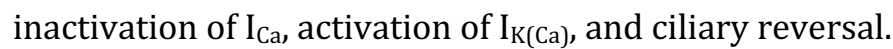

Figures 4E1 and 4E2 show the fits for the cell shown in Figs. 4A and 4B. Consistently with previous voltage-clamp measurements (Oertel et al., 1977), the calcium current is transient and peaks at about $\sim 3.5 \mathrm{nA}$ (Fig. 4E3). A residual current remains, so that calcium concentration remains high during stimulation (Fig. 4E4). This is consistent with the fact that ciliary reversal can last for many seconds when the membrane is depolarized (Machemer, 1974). With large currents, calcium concentration raises to about $22 \mu \mathrm{M}$, similar to previous estimations.

The voltage-gated potassium current is delayed relative to the calcium current, and the calciumactivated $\mathrm{K}^{+}$current raises more slowly and is only dominant during repolarization (Fig. 4E3), with a maximum of $0.65 \mathrm{nA}$. This is consistent with previous studies of that current (Satow and Kung, 1980). Figure $4 \mathrm{~F}$ shows the three different currents during the action potential shown in Fig. 4F. As previously argued, the calcium-activated $\mathrm{K}^{+}$current has a small contribution to the early current (Oertel et al., 1977).

299 Over the $n=18$ cells, we find that the calcium current has half-activation voltage $V_{C a}=-1 \mathrm{mV}(0 \pm 6$ $\mathrm{mV})$, activation slope $k=4.3 \mathrm{mV}(4.3 \pm 1 \mathrm{mV})$ and time constant about $0.9 \mathrm{~ms}(0.9 \pm 0.4 \mathrm{~ms})$ (Fig. 4G). Estimated conductance is not well constrained and often very large. This is presumably because the peak current is mainly determined by the inactivation properties, and therefore the conductance parameter is not well constrained. Half-inactivation occurs at about $K_{C a}=3.7 \mu \mathrm{M}\left(\log _{10}\left(\mathrm{~K}_{\mathrm{Ca}}\right.\right.$ in $\left.\mathrm{M}\right)=-$ $5.4 \pm 0.2$ ). This is close to patch-clamp measurements on cardiac L-type calcium channels (Höfer et al., 1997). The fitted models have about 4 binding sites ( $4.4 \pm 1.7$ ), larger than previous models (Eckert and Chad, 1984; Standen and Stanfield, 1982) (which have a single site but were not constrained by Paramecium data). Calcium decays with a median time constant of $130 \mathrm{~ms}$ and the pump operating near rest has a median maximum rate of $87 \mu \mathrm{M} / \mathrm{s}$.

The delayed rectifier current has similar fitted parameters as in deciliated cells (median $k_{K d}=4.9 \mathrm{mV}$, $\tau_{K d}=4.5 \mathrm{~ms}$ ), except half-activation tends to be lower (median $V_{K d}=4 \mathrm{mV}$ ) - this might be because the responses are essentially below $V_{\mathrm{Kd}}$, in the unsaturated part of the activation curve. The calciumactivated $\mathrm{K}^{+}$current $\mathrm{I}_{\mathrm{K}(\mathrm{Ca})}$ has low affinity $\left(\log _{10}\left(\mathrm{~K}_{\mathrm{KCa}}\right.\right.$ in $\left.\left.\mathrm{M}\right)=-4.2 \pm 0.7\right)$. This is consistent with the observation that in voltage-clamp, this current keeps on increasing for at least one second (Satow and Kung, 1980). There are about $\mathrm{n}_{\mathrm{KCa}}=3$ binding sites is $(3.5 \pm 2.3)$.

Finally, cilia revert at about $2.4 \mu \mathrm{M}\left(\log _{10}\left(\mathrm{~K}_{\text {motor }}\right.\right.$ in $\left.\left.\mathrm{M}\right)=-5.9 \pm 0.8\right)$. This is close to measurements with triton-permeabilized cells, reporting about $1 \mu \mathrm{M}$. We note that this and other concentration parameters depend on the estimation of intraciliary volume, which is approximate. 


\section{Swimming and turning}

We now examine how Paramecium swims and turns, before coupling the electrophysiological model with swimming motion.

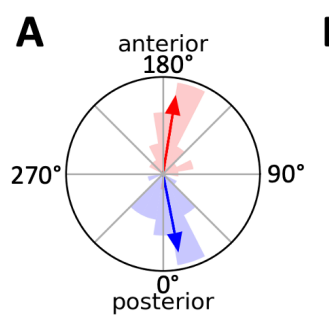

B

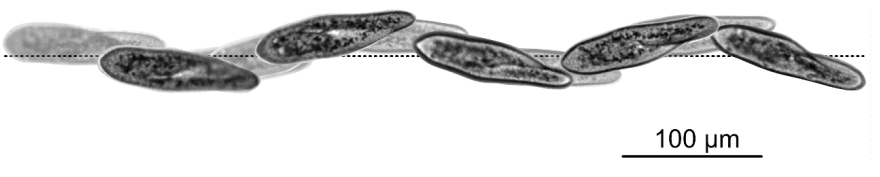

C

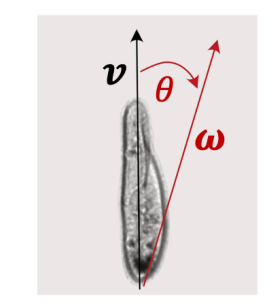

D
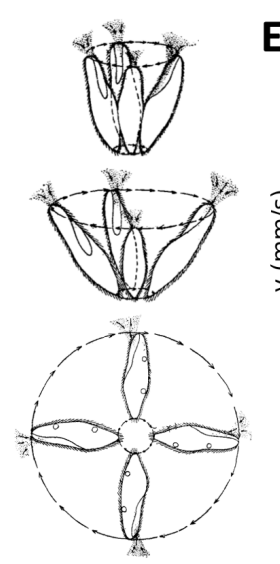

$\mathbf{F}$

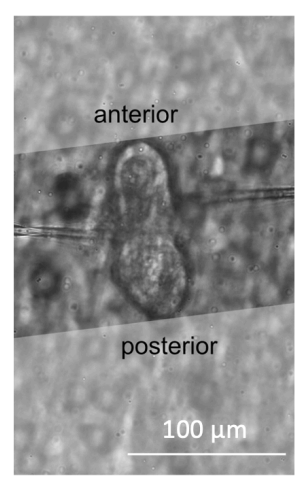

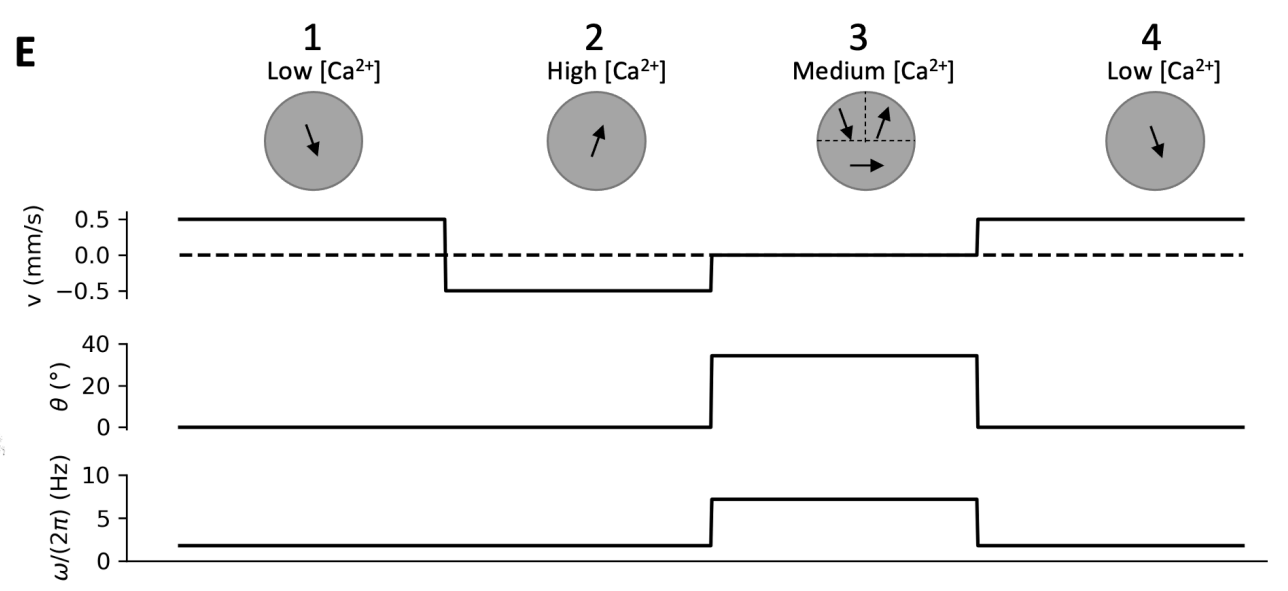
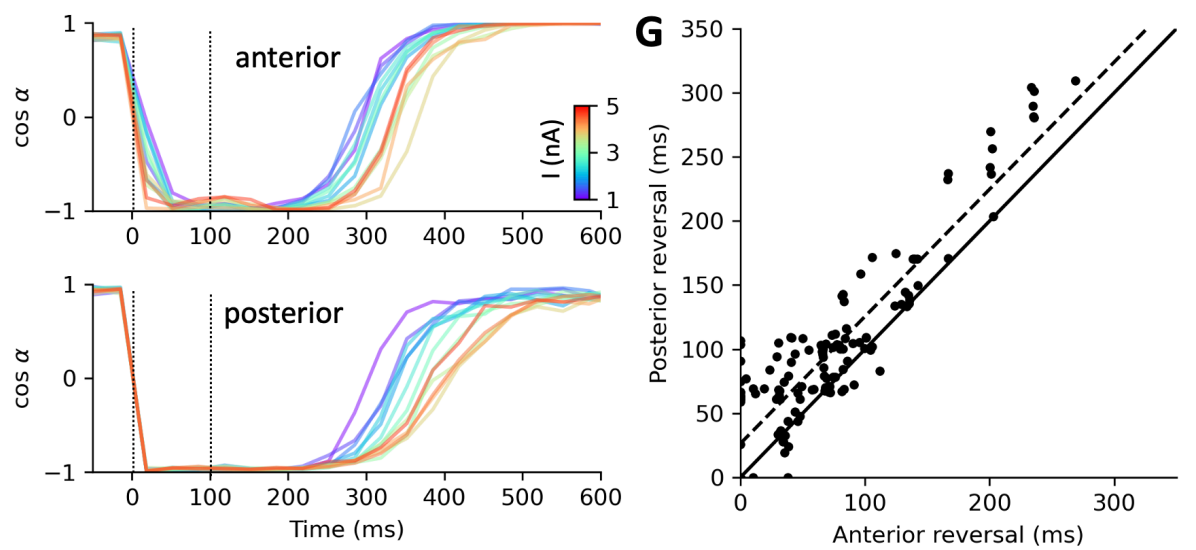

Figure 5. Swimming and turning. A, Direction of fluid motion during forward swimming (blue) and backward swimming (red), relative to the anteroposterior axis. Averages are shown by arrows. B, Example of helicoidal motion of Paramecium, with the oral groove facing the axis. Highlighted frames are spaced by $750 \mathrm{~ms}$. C, The translational velocity vector $\boldsymbol{v}$ is oriented along the anteroposterior axis. The rotation vector $\boldsymbol{\omega}$ is in the dorsoventral plane (including the oral groove), making an angle $\theta$ with the anteroposterior axis. D, Rotating movement at the end of avoiding reactions of increasing strength (Jennings, 1904). E, Calculation of kinematic parameters $v, \theta$ and $\omega$ in a spherical model of radius $60 \mu \mathrm{m}$, during successive phases of the avoiding reaction. First column: cilia beat to the rear and right, producing an axisymmetric force field pushing the organism forward while spinning around its axis. Local force amplitude is adjusted for a velocity of $500 \mu \mathrm{m} / \mathrm{s}$. Second column: cilia revert and now beat to the front and right, pushing the organism backward. Third column: anterior left cilia revert back to the initial direction while anterior right cilia still beat towards the front, and posterior cilia partially revert, beating to the right. Translational velocity is now 0 and the rotation axis tilts to about $34^{\circ}$. Spinning speed $\omega$ also increases by a factor four. Fourth column: all cilia revert back to the initial beating direction. F, Measurement of fluid velocity in a sample cell beyond the anterior end (top) and beyond 
the posterior end (bottom), in response to positive current pulses (1-5 nA), relative to the anteroposterior axis. $G$, Over $n=9$ cells, the direction of posterior motion reverts back about 30 ms after anterior fluid motion (dashed line: linear regression). Reversal duration is calculated as the time when $\cos (\alpha)$ crosses 0 , relative to the pulse end time.

Before stimulation, the flow produced by the cilia is directed towards the posterior end, about $11^{\circ}$ to the right (Fig. 5A, blue). This should produce a forward left spiraling movement, as documented from observations of free swimming (Jennings, 1904; Machemer, 1972). During a pulse that triggers an action potential, the flow is directed towards the anterior end, about $9^{\circ}$ to the right (Fig. 5A, red). This would make the cell swim backward, also spiraling to the left. An example of this spiraling motion is shown on Fig. 5B. The oral groove faces the spiral axis (Bullington, 1930; Herbert S. Jennings, 1899), which means that the rotational velocity vector $\boldsymbol{\omega}$ is tilted from the main axis towards the oral side by an angle $\theta$ (in the median plane; Fig. 5C). In freely swimming paramecia, we found that $\theta \approx 13^{\circ}\left( \pm 6.4^{\circ}\right)$ and the rotation speed $\|\boldsymbol{\omega}\|$ is about 1 cycle/s (1.03 \pm 0.2 cycle/s) (see Methods, Behavioral measurements and Behavioral analysis).

How does Paramecium turn? A directional change can occur if the angle $\theta$ changes. According to Jennings (1904), $\theta$ increases during the avoiding reaction, in relation with stimulus strength (Fig. 5D). To understand the relationship between ciliary beating patterns and kinematic parameters, in particular $\theta$, we examine a spherical model of radius $60 \mu \mathrm{m}$ (Fig. 5E), for which we can use analytical formula relating forces and motion (see Methods, Hydrodynamic model). The fluid produces local forces opposite to the direction of ciliary beating, and the total force and torque map linearly to the translational velocity vector $\mathbf{v}$ and the rotational velocity vector $\boldsymbol{\omega}$ in the cell coordinate system (Lauga and Powers, 2009).

At rest (low calcium concentration), cilia beat towards the rear, slightly to the right (Fig. 5E, first column), so that the fluid produces a force towards the front, slightly to the left. If the direction of ciliary beating is identical everywhere in spherical coordinates (that is, in terms of the cardinal directions North/South/East/West, Fig. 9), then the force field over the sphere is symmetrical with respect to the main axis. This makes both the total force and the total torque align with the main (antero-posterior) axis, and therefore $\mathbf{v}$ and $\boldsymbol{\omega}$ are also aligned with that axis, that is, $\theta=0$. The organism then moves forward, with a spinning movement around the axis. We adjust the force so that the velocity is $500 \mu \mathrm{m} / \mathrm{s}$, which makes the sphere spin at about $1.8 \mathrm{~Hz}$. Upon stimulation, when calcium concentration is high, cilia revert and beat forward (Fig. 5E, second column), making the organism move backward.

Thus, the organism cannot turn unless there is some asymmetry in the ciliary beating pattern. Machemer (1969) and Párducz (1967) observed that during the turning phase, anterior and posterior cilia beat in different directions; Jennings (1904) observed that left and right anterior cilia beat in different directions, where "left" and "right" are relative to the oral groove. In Fig. 5E (third column), we examine what happens if cilia beat in a swirling pattern around the oral groove: the left anterior cilia beating backward, the right anterior cilia beating forward, and the posterior cilia beating to the right. This corresponds to what would happen near the calcium concentration threshold for global ciliary reversal, if cilia revert back first in the left anterior part, then in the posterior part, then in the right anterior part. The swirling pattern suggests that the cell is going to turn around an axis tilted from the main axis, in the plane separating the left and right sides, and this can be confirmed analytically. In this configuration, the sphere does not move along the main axis ( $v=0 \mathrm{~mm} / \mathrm{s}$ ), because the net force along that axis is null, but it turns along an axis tilted by $\theta \approx 34^{\circ}$ from the main axis. The sphere also spins about 4 times faster $(\omega /(2 \pi) \approx 7.2 \mathrm{~Hz})$. It returns to moving forward when all cilia revert back (Fig. 5E, fourth column). 
This pattern of ciliary reversal is suggested on Fig. 1C. However, given that the particle flow was measured on a plane $\sim 30 \mu \mathrm{m}$ above the cell, that the cell could take different shapes and that the position of the oral groove was often difficult to estimate, it was generally not possible to determine the precise pattern of ciliary reversal empirically. Nevertheless, it is possible to demonstrate that cilia revert asynchronously. We measured particle flow separately in two regions of the field, beyond the anterior end, and beyond the posterior end (Fig. 5F). This was only possible for 9 cells, where video quality was sufficient (see Methods, Particle image velocimetry). We measured the mean angle $\alpha$ of the flow field during stimulation with current pulses between $1 \mathrm{nA}$ and $5 \mathrm{nA}$; weak pulses were not included because particle density tended to be lower due to sedimentation (weak pulses were recorded after strong pulses).

Figure 5F shows in one cell that posterior cilia (bottom) revert back after anterior cilia (top). Thus, before stimulation, when calcium concentration is low, anterior and posterior cilia beat in the same direction. Beating direction is also spatially homogeneous during stimulation, when calcium concentration is high. However, after stimulation, anterior and posterior cilia beat in different directions for a short period. This was a reproducible finding across the 9 cells: the posterior side tends to revert back slightly later than the anterior side (Fig. $5 \mathrm{G})\left(\mathrm{p}=7.10^{-17}\right.$, one-tailed Wilcoxon test), with a mean delay of 26 ms (s.d. 29 ms). This confirms Párducz' observations (Párducz, 1967), which were based on electron microscopy of cells fixed during the avoiding reaction.

\section{Modeling the avoiding reaction}

We now use this analysis to connect the electrophysiological model with motion of the organism. As illustrated in Fig. 5C, we will assume that the translational velocity vector $\mathbf{v}$ is aligned with the main axis, so that it is fully parameterized by the velocity $v$, and that the rotation vector $\boldsymbol{\omega}$ lies in the plane of the oral groove, so that it is parameterized by its angle $\theta$ relative to the main axis and the spinning speed $\omega$. We assume that all three kinematic parameters $(v, \theta, \omega)$ are functions of intraciliary calcium concentration [Ca ${ }^{2+}$ (Fig. 6A-C) (See Methods, Electromotor coupling, equations (21-23)).

We model velocity as a Hill function of [ $\mathrm{Ca}^{2+}$, with threshold equal to the reversal threshold $K_{C a}$ and $n$ $=2$ sites, linearly scaled to match the maximum positive and negative velocities measured empirically (Fig. 6A; equation (21)). In freely swimming paramecia, we observed that the median velocity was 472 $\mu \mathrm{m} / \mathrm{s}(521 \pm 285 \mu \mathrm{m} / \mathrm{s})$ for forward swimming and $370 \mathrm{~mm} / \mathrm{s}(411 \pm 200 \mu \mathrm{m} / \mathrm{s})$ for backward swimming. Thus, in the model, we simply set both forward and backward maximum velocity at $v=$ $\pm 500 \mu \mathrm{m} / \mathrm{s}$. Figure $6 \mathrm{~A}$ shows the resulting function for the cell shown in Fig. 4A-F.

We model both $\theta$ and $\omega$ as bell functions of [ $\mathrm{Ca}^{2+}$ ] (Fig. 6B, C; equations (22-23); see Methods, Electromotor coupling), peaking when $\left[\mathrm{Ca}^{2+}\right]$ is near the global ciliary reversal threshold $K_{\text {motor }}$, as suggested by our analysis of the spherical model (Fig. 5E). The minimum angle is taken from measurements of trajectories of freely swimming paramecia $\left(\theta \approx 13^{\circ}\right)$. For the maximum angle, we choose $\theta=90^{\circ}$ to account for the strongest avoiding reactions observed by Jennings (Fig. 5D), corresponding to a rotation normal to the main axis. The minimum spinning speed is based on measurements ( $\omega \approx 1 \mathrm{cycle} / \mathrm{s}$ ), and the maximum spinning speed is set to 4 times the minimum, as in the spherical model (Fig. 5D). 

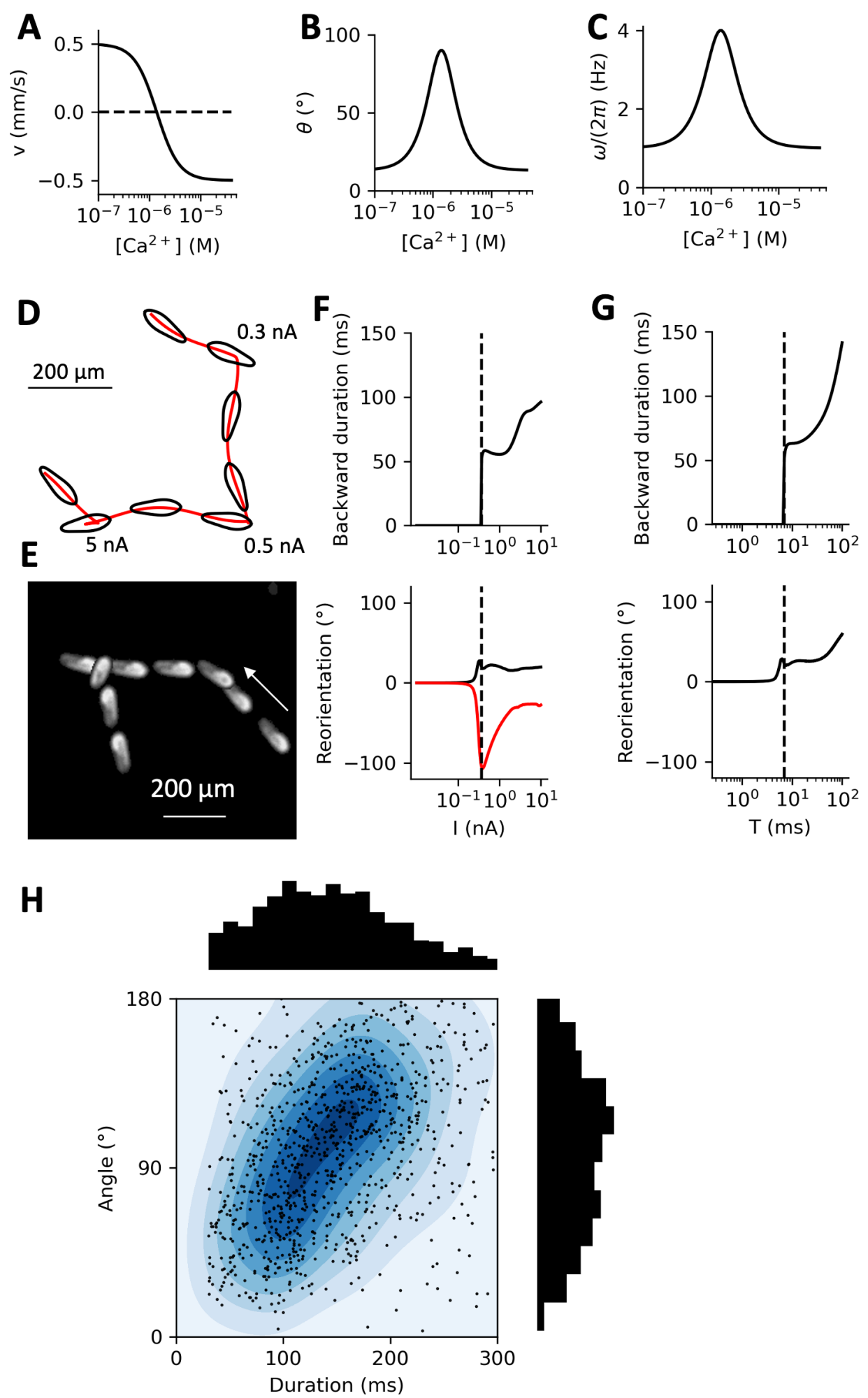

Figure 6. Simulation of the avoiding reaction. A, Velocity $v$ as a function of intraciliary calcium concentration $\left[\mathrm{Ca}^{2+}\right]$ in the model. B, Angle $\theta$ of the rotation axis as a function of $\left[\mathrm{Ca}^{2+}\right]$ in the model. C, Spinning speed $\omega$ as a function of $\left[\mathrm{Ca}^{2+}\right]$ in the model. Angle and spinning speed increase at intermediate $\mathrm{Ca}^{2+}$ concentration, as implied by the spherical model in Fig. 5E. D, Simulated model trajectory with three $2 \mathrm{~ms}$ current pulse stimulations of increasing amplitude. Images are shown at $400 \mathrm{~ms}$ intervals. Without stimulation, the organism swims in spiral. A very small stimulation deviates the trajectory. Stronger stimulations produce avoiding reactions, with backward swimming and turning. E, Example of an observed Paramecium trajectory showing 
are shown at $400 \mathrm{~ms}$ intervals, starting on the right. F, Backward swimming duration and reorientation angle as a function of current amplitude for 2 ms pulses. Red and black curves show results for the same model but different initial positions of the oral groove, differing by a quarter of a cycle. G, Backward swimming duration and reorientation angle as a function of current pulse duration $T$ with $100 \mathrm{pA}$ amplitude. $\mathrm{H}$, Reorientation angle vs. backward swimming duration in $n=1138$ spontaneous avoiding reactions of Paramecium, showing a positive correlation (linear regression $r=0.2, p \approx 10^{-11}$ ). About $15 \%$ of data points are not represented (larger angle or duration).

In this way, we obtain a model in which all kinematic variables are coupled to the electrophysiological model. We can then calculate organism motion from these variables, and thereby simulate behavior in an environment (see Methods, Kinematics). In the following, the model of one particular cell is chosen for illustration, the same cell as in Fig. 4.

We first examine the trajectory of a model stimulated by 2 ms currents of varying amplitude (Fig. 6D) (see Methods, Behavioral scenarios). Without stimulation, the organism swims in a helicoidal path. With a small stimulation amplitude $(0.3 \mathrm{nA})$, the organism changes direction without swimming backward. At larger amplitude $(0.5 \mathrm{nA})$, the organism swims backward for a very short time then turns and swims forward. When the amplitude is increased ( $5 \mathrm{nA})$, backward swimming is more noticeable. Directional changes without backward swimming do occur in freely swimming paramecia, as illustrated on Fig. 6E: the organism first changes direction without swimming backward, then does an avoiding reaction.

In more detail (Fig. 6F, top), we observe that the cell swims backward when current intensity exceeds a threshold (here $372 \mathrm{pA}$ ), then backward swimming duration tends to increase with intensity. The reorientation angle following the stimulation changes continuously with stimulation strength, but in a complex way (Fig. 6F, bottom). In particular, small stimulations can trigger large turns without backward swimming. In addition, the directional change depends on the initial position of the oral groove: the red and black curves of Fig. $6 \mathrm{~F}$ (bottom) correspond to the same cell but an oral groove position (spinning angle) differing by a quarter of a cycle.

The characteristics of the avoiding reaction also depend on stimulus duration (Fig. 6G). If the pulse amplitude is fixed $(\mathrm{I}=0.1 \mathrm{nA})$ and its duration is increased, then the duration of backward swimming increases (Fig. 6G, top), and reorientation angle tends to increase for large durations but is not monotonous near threshold (Fig. 6G, bottom).

When we examine spontaneous avoiding reactions of freely swimming paramecia, we find that both backward swimming duration and reorientation angle vary broadly $\left(156 \pm 81 \mathrm{~ms}\right.$ and $114 \pm 66^{\circ}$, respectively) (Fig. $6 \mathrm{H}$ ), and there is a small although highly significant correlation (linear regression, $\mathrm{r}=0.2, \mathrm{p}=10^{-11}$ ). Thus, backward swimming duration and reorientation angle are variable and not deterministically related.

\section{A closed-loop behavioral model of Paramecium}

We now use the model to describe how the interaction between organism and environment gives rise to behavior (see Methods, Behavioral scenarios). 

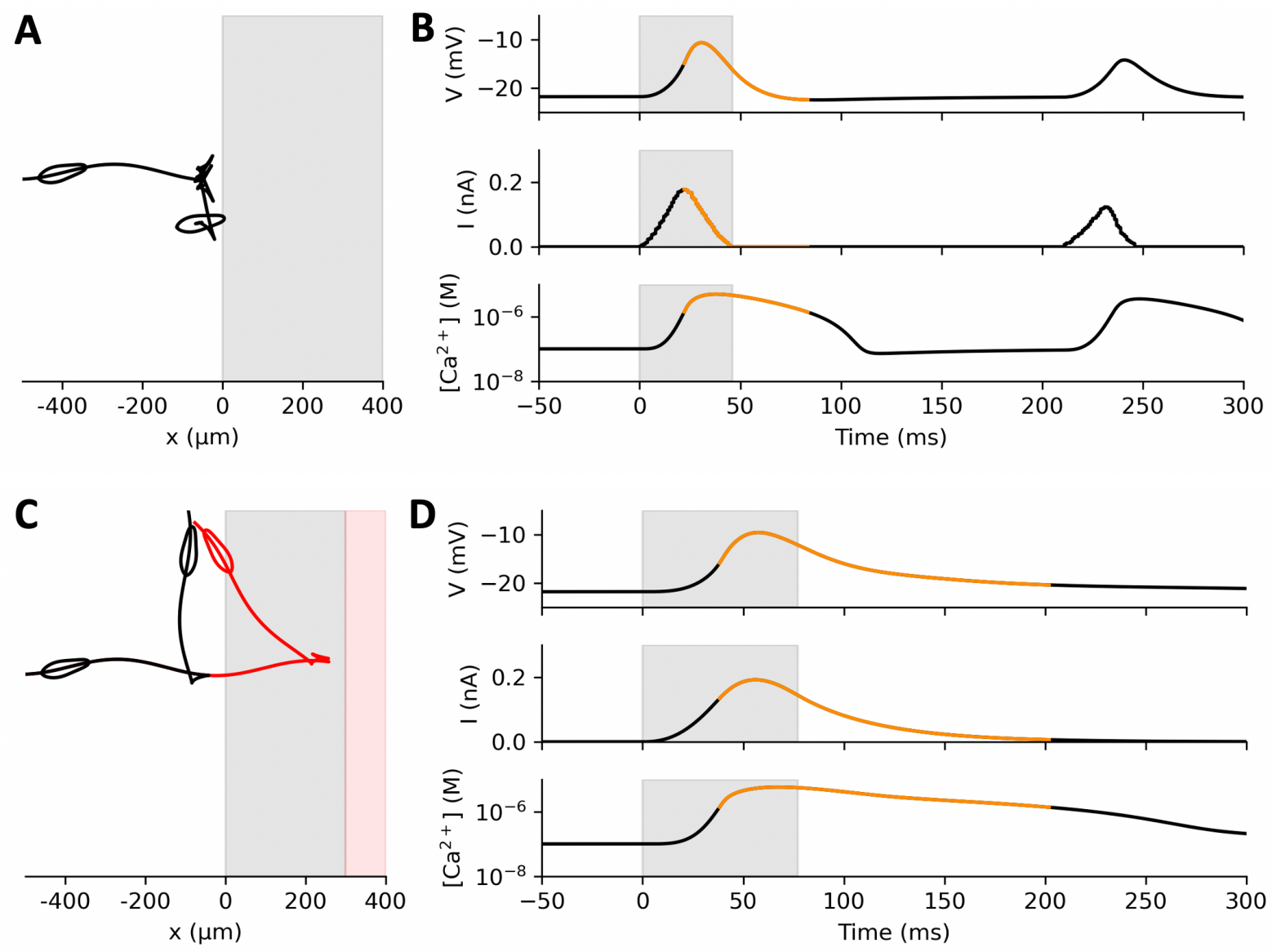

Figure 7. Interaction of a model Paramecium with a generic stimulus, modelled as a positive current proportional to the cell area within the stimulus area. A, Trajectory of the model doing several avoiding reactions against the stimulus. B, Membrane potential (top), stimulus current (middle) and intraciliary calcium concentration (bottom) at the first contact. Contact occurs at the boundary with the shaded region. Orange curves indicate backward swimming. Several weak avoiding reactions occur in succession. C, Trajectory of the model where sensory transduction has a 50 ms activation/deactivation time constant. In red, the stimulus is placed $300 \mu \mathrm{m}$ further away. D, Same as B, for the black trajectory in $C$. The stimulus current lasts longer and peaks after the organism has started reacting, resulting in a stronger avoiding reaction.

First, we consider an organism swimming towards a generic object, which triggers a depolarizing current when in contact with the membrane (for example a chemical substance, or hot water) (Fig. 7). Thus, we simply consider that the stimulus current is proportional to the surface area in contact with the stimulus (see Methods, Sensory transduction). In contrast with previous situations, the stimulus is not pre-determined but depends on behavior. As Dewey pointed out (1896), "the motor response determines the stimulus, just as truly as sensory stimulus determines the movement.". When the organism touches the object, a current is triggered, which depolarizes the membrane (Fig. 7A, 7B). As the cell swims into the object, the current increases until an action potential is triggered. The cell then swims backward, moves out from the object and the current stops. Thus, the sensory current is necessarily small and short, because the organism withdraws as soon as the current reaches threshold. This results in a small avoiding reaction, and the organism bumps again repetitively against the object until it finally escapes (Movie 1).

Larger movements can be obtained if sensory transduction has slower kinetics (Figs. 7C, D and Movie 2). Here, the sensory current follows the stimulation with a time constant of $40 \mathrm{~ms}$, modelled with first order kinetics (to simplify, the spatial spread of channels is not modeled; equation (24)). In this case, 
the sensory current keeps on increasing (slightly) after the organism has started swimming backward and it lasts longer. This results in a larger avoiding reaction.

499 Although the model is deterministic, the directional change can be described as pseudo-random. If the object is moved $300 \mu \mathrm{m}$ further (Fig. 7C), then the organism escapes with a larger angle. This is because the organism spins while swimming, so that its oral groove takes a different position when it touches the object.

503 We now consider that the object is a disk in a square environment (Fig. 8A and Movie 4). To avoid 504 boundary effects, we consider that the environment has the topology of a torus (paramecia escaping 505 to the left reappear to the right). To account for spontaneous avoiding reactions (occurring at a rate of 506 about $0.2 \mathrm{~Hz}$ in our behavioral measurements), we added a noisy current to the membrane equation. 507 The paramecia are modeled as in Figs. 7C and D, with slow transduction. Fig. 8A shows 100 trajectories 508 starting from random positions and simulated for $20 \mathrm{~s}$. The proportion of paramecia inside the disk 509 decreases from 19 to $7 \%$. Thus, the disk acts as a repelling stimulus. Occasionally, a paramecium gives 510 an avoiding reaction against the boundary, makes a large turn and swims backward into the disk. In 511 this case, it continues being stimulated and swims backward through the disk until it escapes. This 512 peculiar behavior might be avoided if currents of opposite polarity were triggered when stimulating 513 the rear, as is the case for thermal stimuli (Tominaga and Naitoh, 1992) and some chemical substances 514 (Oami, 1996). Traces shown in Fig. 8A show the membrane potential $V$, the stimulus current $I$ and the 515 calcium concentration $\left[\mathrm{Ca}^{2+}\right]$ for the trajectory shown in red, where we can distinguish a number of 516 spontaneous avoiding reactions and one avoiding reaction against the disk.

517 Next, we examine how an object can act as an attractive stimulus (Fig. 8B and Movie 5). This behavior 518 can be obtained if the cell responds to a hyperpolarizing stimulus with adaptation, so that a 519 depolarization is triggered when the stimulus stops. A simple model that exhibits this behavior is one 520 where the stimulus triggers currents through two pathways with different kinetics: a hyperpolarizing 521 current with fast kinetics, and a depolarizing current of equal magnitude with slow kinetics (equations 522 (25-27)). In this way, a transient hyperpolarizing current is triggered when the stimulus switches on, and a transient depolarizing current is triggered when the stimulus switches off. This is shown on Fig. 8B. The proportion of paramecia inside the disk increases with time (from $15 \%$ to $45 \%$ in 20 s). It can be seen that paramecia tend to aggregate inside the disk, mostly near the boundary. This is due to the small avoiding reactions but also to the curvature of the disk. 

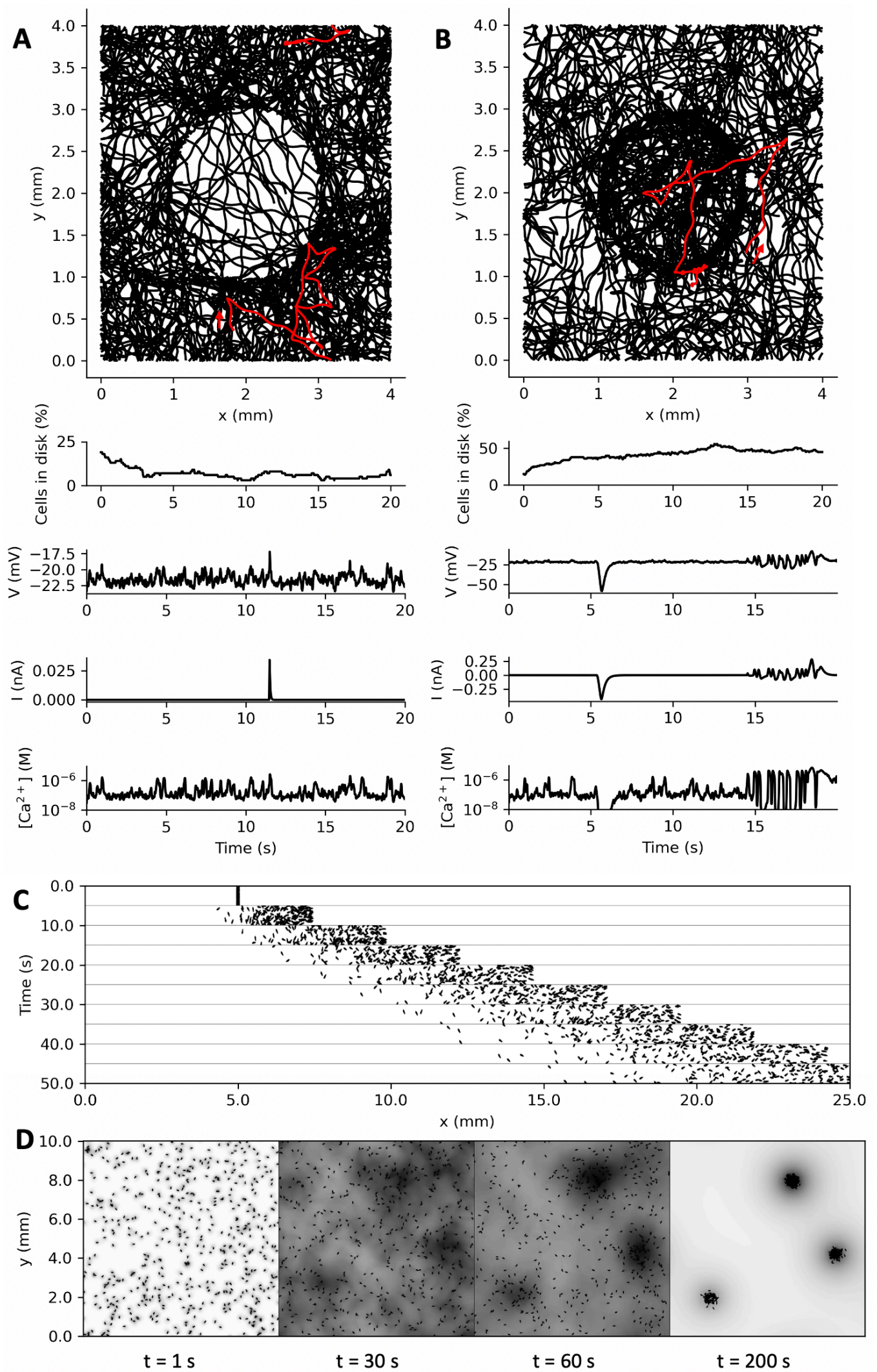

528 Figure 8. Closed-loop behavior of the Paramecium model. A, Top: trajectories of 100 models swimming for 20

529 s in a torus with a depolarizing circular stimulus, modelled as in Fig. 7C. The proportion of cells in the disk 530 quickly decays (below). Membrane potential, stimulus current, and intraciliary calcium concentration are 531 shown for the trajectory highlighted in red, which does an avoiding reaction against the disk after a number 532 of spontaneous avoiding reactions. B, 100 model trajectories with a circular stimulus triggering an adapting 
hyperpolarizing current. Organisms tend to make avoiding reactions on the inner boundary of the disk. The proportion of cells in the disk increases over time. The highlighted trajectory enters the disk around $t=5 \mathrm{~s}$ with a large hyperpolarization, then displays several avoiding reactions against the boundary of the disk before exiting. C, Paramecia swimming in a linear stimulus gradient, modelled as in B. The position of 200 cells starting at position $x=5 \mathrm{~mm}$ is displayed every $5 \mathrm{~s}$. D. Collective behavior in model paramecia induced by breathing and chemosensitivity. $\mathrm{CO}_{2}$ produced by cells is displayed in shades of grey (normalized to the spatial peak), and diffusion is simulated. $\mathrm{CO}_{2}$ concentration represents an attracting stimulus modelled as in $\mathrm{B}$ and $\mathrm{C}$.

Using the same model, we then place the paramecia in a linear stimulus gradient (Fig. 8C); the environment is toric in the transversal dimension (up and down boundaries are glued). We place all paramecia at the same initial position, with random orientations. The population then rapidly ascends the gradient. This occurs because a depolarizing current is produced when the stimulus decreases, triggering an avoiding reaction. This behavior has been observed in Paramecium with thermal gradients (H. S. Jennings, 1906; Mendelssohn, 1895), and shares similarities with bacterial chemotaxis (Berg, 1975).

Finally, we show an example of collective behavior (Fig. 8D). Paramecium produces $\mathrm{CO}_{2}$ by breathing, which acidifies its medium, and it is attracted by weak acids (Dryl, 1973; Houten, 1978; H. S. Jennings, 1899). As a result, it can form aggregates, for example around a source of food or at the bottom of a depression slide (H. S. Jennings, 1899). We simulate the production of $\mathrm{CO}_{2}$ by paramecia and its diffusion in the medium (a square with torus topology), together with sensitivity to $\mathrm{CO}_{2}$ modeled in the same way as in Fig. 8B and C. $\mathrm{CO}_{2}$ concentration is represented in Fig. 8D as grey shades, after normalization. In this simulation, paramecia progressively form aggregates.

\section{Discussion}

\section{Summary}

557 We have built an integrative model of Paramecium that combines electrophysiology and motility. The 558 model is informed by previous experimental literature and constrained by specific electrophysiology 559 and behavioral experiments. It models mainly the action potential and its coupling with kinematic 560 parameters, which allows it to be simulated as a model of autonomous behavior in various 561 environments.

562 The electrophysiological model was built by model fitting to current clamp data, with calciumdependent properties indirectly constrained by ciliary reversal data. This method recovered properties of individual currents compatible with previous measurements obtained by different means. For example, the calcium threshold for ciliary reversal was estimated to be $\sim 2 \mu \mathrm{M}$, the same order of magnitude as measured by varying extracellular calcium concentration in Paramecium with permeabilized membranes (Naitoh and Kaneko, 1972). This is notable since calcium was not measured but only inferred from electrophysiology (indirectly through the estimation of the calcium current by the fitting procedure). The fitting procedure also determined that the calcium-dependent $\mathrm{K}^{+}$current is small during the action potential, as previously determined with voltage-clamp experiments (Oertel et al., 1977), but dominant after stimulation. The magnitude and time scale of calcium currents estimated by fitting were also compatible with voltage-clamp measurements (Oertel et al., 1977). Quantitative fitting allowed us to estimate additionally the calcium inactivation threshold $(\sim 3 \mu \mathrm{M})$ and the number

574 of inactivation sites $(\sim 4)$.

575 By measuring ciliary induced flows during action potentials, we found that ciliary reversal is not 576 synchronous across the cell, confirming previous observations obtained with electron microscopy 
(Párducz, 1967). We showed with a simple hydrodynamic model that asynchronous ciliary reversal allows the organism to turn, namely if the order of ciliary reversal follows a swirling pattern around the oral groove. From these findings, we built a phenomenological model of the coupling between calcium concentration and the main kinetic parameters, constrained with behavioral measurements (speed, angle).

The integrated model shows helicoidal swimming with graded avoiding reactions, where backward duration swimming and reorientation angle increase with stimulus strength or duration. As observed in spontaneous behavior, the model can also slightly reorient without swimming backward.

Behavior of the autonomous model is more complex than stimulus-response experiments, because the relation between sensory stimulus and motor response is circular. In particular, we noticed that the interaction with an object (e.g. a chemical substance) critically depends on the properties of sensory transduction. For example, efficient avoidance of the object requires persistent stimulation, e.g. with slow sensory activation/deactivation. Furthermore, sensory adaptation to a hyperpolarizing stimulus makes the stimulus attractive. This can allow the model to follow a stimulus gradient. Finally, collective behavior can arise if organisms are sensitive to a substance that they produce. In summary, relatively complex behavior can be generated by the interaction of this simple "swimming neuron" with its environment.

\section{Limitations}

This work has many limitations. First, ionic currents were measured simultaneously rather than in isolation, although we could isolate $I_{K d}$ by deciliation. This was partly for technical reasons (one cannot measure $\mathrm{I}_{\mathrm{K}(\mathrm{Ca})}$ while blocking the calcium current), and partly to ensure a global fit of the entire model to the action potential. Nonetheless, current overlap may cause difficulties for model fitting. For this reason, we strived to choose the simplest models that captured the phenomenology.

A second limitation is that calcium was not directly measured. Instead, it was indirectly constrained by several observed phenomena: calcium-dependent inactivation of $\mathrm{I}_{\mathrm{Ca}}$, calcium-dependent activation

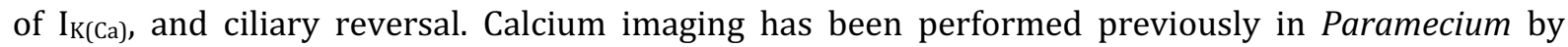
pressure injection of a calcium indicator, in other contexts (Iwadate et al., 1997; Iwadate and Kikuyama, 2001; Klauke and Plattner, 1997). However, it is technically challenging to perform quantitative time-resolved measurements of ciliary calcium, because the cilia represent a small fraction of the total volume $(2-3 \%)$ and beat at about $20 \mathrm{~Hz}$.

Related to this limitation, our estimates of calcium-dependent parameters, for example the ciliary reversal threshold, depend on an estimate of the effective ciliary volume. Compared to our estimate, based on electron microscopy measurements, this effective volume may be reduced by crowding, or increased by fast buffering. Changing this parameter results in proportional changes in concentration parameters. However, the fact that the fitted ciliary reversal threshold is close to the threshold measured on permeabilized Paramecium suggests that our estimate (also used in (Oertel et al., 1977)) was reasonable.

615 Another limitation is we did not measure ciliary beating directly, but rather its effect on the fluid. This 616 was motivated by the fact that we were interested primarily in the movement induced by ciliary 617 beating, as well as by technical reasons. In future work, high speed imaging of ciliary beating could be 618 used to determine the spatial pattern of ciliary reversal with higher precision, although contractions 619 of the cell may complicate the analysis. 
Because of these technical limitations, our model of electromotor coupling was highly simplified, restricted to a phenomenological relation between calcium concentration and three kinetic parameters. It could also be that this relation is not instantaneous, involving more indirect pathways.

Finally, we considered only generic rather than biophysical models of sensory transduction, and we did not consider mechanical or hydrodynamic interactions with objects (Berke et al., 2008; Jana et al., 2015; Ohmura et al., 2018). More generally, the behavioral repertoire of Paramecium includes other aspects that we did not attempt to model, such as the escape reaction (Machemer, 1974; Roesle, 1903), contractions (Nakaoka and Machemer, 1990), trichocyst discharge (Hamel et al., 2011; Knoll et al., 1991) and gravitaxis (Jensen, 1893).

\section{How Paramecium turns}

631 Since our model directly couples calcium concentration to kinetic parameters, it is not tied to any specific hypothesis about the ciliary beating pattern. However, turning is only possible if ciliary reversal is asynchronous, leading to a strongly asymmetrical ciliary beating pattern, otherwise the action potential would only trigger a back-and-forth movement in the direction of the main axis. We have shown that one possibility, compatible with the observed movement, is that cilia beat in a swirling pattern around the oral groove.

It is known that there is some structural and molecular heterogeneity between cilia, in particular between locomotor and oral cilia (Aubusson-Fleury et al., 2015). Whereas basal bodies are regularly placed on the dorsal side, they are spatially arranged on the ventral side with a characteristic pattern around the oral groove. The beating frequency during helicoidal swimming is also spatially heterogeneous (Jung et al., 2014), and tail cilia are also known to be immobile (Machemer and Machemer-Röhnisch, 1984). Ciliary heterogeneity is likely to be more complex than a distinction between oral and locomotor cilia, because when Paramecium is cut in two pieces below the oral groove, both pieces can turn in a similar way (Jennings and Jamieson, 1902). Such heterogeneity is likely a general feature of motile microorganisms, some of which can exhibit complex gaits (Wan, 2020).

Machemer (1969) and Párducz (1967) described an asynchrony between anterior and posterior ciliary reversal, with anterior cilia returning to their initial beating direction before posterior cilia, which we confirmed with our PIV measurements. However, this is not sufficient to produce turning: if both anterior and posterior beating patterns are axisymmetric, any combination of them would still produce movement along the main axis. Jennings mentions that there is also a reversal asynchrony between the anterior left and anterior right cilia, such that all anterior cilia transiently beat towards the oral groove during the avoiding reaction (Jennings, 1904). More detailed investigation is necessary to clarify this question.

655 Physiologically, asynchronous ciliary reversal can be due to differential calcium sensitivity, that is, the calcium threshold for reversal might vary across cilia. This is the implicit assumption of our model. It could also be that there are differences in calcium entry or removal across cilia (e.g. a gradient of calcium channel expression, or calcium pumps, or calcium buffering molecules). Some studies suggest that cyclic nucleotides may also differentially regulate the reversal threshold (Noguchi et al., 2000, 1991).

\section{Previous models}


We are aware of two previous attempts to model Paramecium's action potential, neither of which was based on quantitative measurements. Hook and Hildebrand (1979) used a calcium channel model with instantaneous transitions, an ohmic current-voltage relation (instead of GHK), an inactivation state accessible only from the closed state, and no voltage-dependent $\mathrm{K}^{+}$channel (only a model of $\mathrm{I}_{\mathrm{K}(\mathrm{Ca}) \text {, }}$ which is not the major $\mathrm{K}^{+}$current). Kunita et al. (2014) used a Hodgkin-Huxley type model with voltage-dependent inactivation of calcium channels, which is not the main inactivation mode of this channel (Brehm et al., 1980), even though the phenomenon exists on a slow timescale (Hennessey and Kung, 1985). The model included two calcium channels (fast and slow), for which there is no electrophysiological support, and their relative activation was given as a function of time after stimulus start (i.e., it is not modeled). The calcium-dependent $\mathrm{K}^{+}$current was not included. Neither electrophysiological model was fitted to data, and neither was coupled to a kinematic model.

\section{Perspectives}

The model could be improved by addressing the technical limitations listed above. In particular, it would be most enlightening to measure calcium concentration in the cilia at high temporal and spatial resolution, although it might require new technical developments. Further investigations should be carried out to understand in detail how Paramecium turns: to measure the spatial pattern of ciliary responses and to determine how this heterogeneity is achieved physiologically.

We have addressed only the avoiding reaction of Paramecium. The modeling effort could be completed by addressing other behavioral aspects, such as the escape reaction (increased ciliary beating speed upon hyperpolarization), which involves distinct hyperpolarization-activated channels (Brette, 2021). Paramecium is sensitive to many sensory modalities, including temperature, various chemical substances, mechanical stimulation, light. Thus, the model should be completed by models of sensory transduction, as well as of mechanical interaction with objects. This would allow us to use the model to investigate the physiological basis of behavior of Paramecium in complex environments.

Finally, this work opens the perspective of addressing complex autonomous behavior in ecological environments, including adaptation, learning and problem solving (Brette, 2021), with a systemic modeling approach.

\section{Materials and Methods}

\section{Paramecium culture and preparation}

694 Cultures of Paramecium tetraurelia were obtained from Éric Meyer, Institut de Biologie, Ecole Normale 695 Supérieure, Paris, France. For electrophysiological experiments (at Institut de la Vision), paramecia were co-cultured with Klebsiella pneumoniae, where each week $1 \mathrm{~mL}$ of culture was reinjected into 5 $\mathrm{mL}$ of Wheat Grass Powder (WGP) buffer supplemented with $1 \mu \mathrm{L}$ of beta-sitosterol. Cultures were kept at room temperature (about $20^{\circ} \mathrm{C}$ ). Cells were harvested in the early stationary growth phase, between 3 and 5 days after feeding them. To wash and concentrate cells for experiments, a droplet of culture (approximately $600 \mu \mathrm{L}$ ) was placed in a narrow neck volumetric flask before adding extracellular solution used for electrophysiology (see below). Due to negative gravitaxis (Naitoh and Eckert, 1972), paramecia tend to accumulate at the top of the solution. Thus, after approximately 10 min, a concentrated population of cells were retrieved from the top of the flask and placed in a 
microcentrifuge tube for at least $3 \mathrm{~h}$ for adaptation (Machemer-Röhnisch and Machemer, 1989; Oka et al., 1986). The tube was shaken before collecting cells to perform an experiment.

The culture method differed slightly for the behavioral measurements with freely swimming paramecia, because these cultures were done in another lab (Laboratoire Jean Perrin). Instead, before an experiment, bacteria were first grown in $5 \mathrm{~mL}$ of WGP for $24 \mathrm{~h}$ at $27^{\circ} \mathrm{C}$, then paramecia were grown by adding $1 \mathrm{~mL}$ of Paramecium culture and $1 \mu \mathrm{L}$ of beta-sitosterol to the bacterized WGP, for $48 \mathrm{~h}$ at $27^{\circ} \mathrm{C}$ in the dark. About $0.4 \mathrm{~mL}$ of cell suspension were then pipetted from the top of the culture tube into $4 \mathrm{~mL}$ of extracellular solution (see Electrophysiology), at least 20 minutes before an experiment.

\section{Swimming pools}

714 Freely swimming paramecia were imaged at room temperature $\left(\sim 25^{\circ} \mathrm{C}\right)$ in square pools of side length $71530 \mathrm{~mm}$ and depth $340 \mu \mathrm{m}$. These were obtained using micromilling and molding techniques. A 716 Plexiglas mold, consisting of a square trench, is first milled with a square end mill of diameter $1 \mathrm{~mm}$ 717 using a CNC micro-milling machine (Minitech, Machinary Corp., USA). Then a liquid mixture of Poly718 DiMethyl Siloxane (PDMS, Sylgard 184, Dow Corning, USA) and its crosslinker (10:1 mass ratio) is 719 poured onto the Plexiglas mold. It is immediately placed in a vacuum chamber for at least $1 \mathrm{~h}$ to remove 720 any air bubbles. Crosslinking of the mixture is then obtained by placing the whole in an oven at $65^{\circ} \mathrm{C}$ 721 for at least $4 \mathrm{~h}$. Finally, the resulting transparent elastomer pool is gently peeled off the mold and put 722 on a microscope glass slide. Prior to any experiment, the pool is exposed to an oxygen plasma for about

7231 min to render the PDMS surface hydrophilic and prevent the trapping of air bubbles.

\section{Behavioral measurements}

For all behavioral experiments, about $500 \mu \mathrm{L}$ of the cell suspension is pipetted into the pool with a concentration of $300-600$ cells/mL. Trajectories are imaged at $50 \mathrm{~Hz}$ with a CMOS camera (Blackfly S BFS-U3-51S5M-C, Flir, USA, 2448x2048 pixels $^{2}, 10$ bits), acquired with its dedicated acquisition software (Spinview, Flir, USA). A high magnification variable zoom lens (MVL12X12Z, Thorlabs) is used and yields a pixel size of $3.81 \mu \mathrm{m}$. The pool is uniformly illuminated with a dark field configuration, by placing $\sim 10 \mathrm{~cm}$ beneath the pool a square LED panel (EFFI-SBL, Effilux, France), on top of which a fully opaque mask is positioned, partially covering the LED panel (typically $1 / 4$ of its surface). The LED panel produces a red light (wavelength $\lambda=625 \mathrm{~nm}$ ) to minimize phototaxis (Iwatsuki and Naitoh, 1983, 1982). Movies of the swimming paramecia are $200 \mathrm{~s}$ long (see Movie 7).

To limit hard drive space, images are stored without their background with lossless compression (TIFF format). The background image is computed by taking for each of its pixels the minimum pixel intensity over the first 100 frames. It is then subtracted from each frame, and pixels with an intensity value below a threshold (automatically computed with the "triangle method", see e.g. (Zack et al., 1977)) are set to 0 .

Trajectories are extracted with the open source tracking software FastTrack (Gallois and Candelier, 2021), and manually inspected for corrections. Briefly, the software fits an ellipse to the cell's shape, and disambiguates front and rear based on the asymmetry of the pixel histogram along the main axis. Trajectories shorter than $1 \mathrm{~s}$ and sequences where the cell is immobile are discarded. Errors in front/rear identification are automatically corrected as follows: when the cell turns by more than $20^{\circ}$ over two successive frames, it is considered an error and the angle is flipped. 
Trajectories with circling motions are also discarded. To this end, we calculate the proportion of the trajectory where the cell turns clockwise versus anti-clockwise (for trajectories longer than $4 \mathrm{~s}$ ). These proportions should be balanced $(0.5)$ for helicoidal trajectories. The trajectory is eliminated if these proportions differ by more than 0.1 from the expectation, with manual confirmation. In total, there were $\mathrm{n}=554$ selected trajectories.

\section{Behavioral analysis}

\section{Analysis of helicoidal trajectories}

754

755

756

757

758

759

We manually selected 20 trajectories presenting clear helicoidal motion in the focal plane from 2 experiments, totaling $121 \mathrm{~s}$. In each helicoidal trajectory, cell orientation $\gamma(t)$ varies periodically with period T. We fitted $\gamma(t)$ to a sinusoidal signal. We found $T=1.02 \pm 0.27 \mathrm{~s}$ (mean \pm s.d.), corresponding to a spinning speed $\omega=\frac{1}{T}=1.03 \pm 0.2 \mathrm{cycle} / \mathrm{s}$ or about $2 \pi / \mathrm{s}$ in radians. The amplitude was $\theta=13 \pm 6.4^{\circ}$, the angle relative to the spiral axis.

\section{Analysis of avoiding reactions}

An avoiding reaction is defined as a portion of trajectory during which the cell swims backward. This backward swimming is detected when the instantaneous motion vector $\mathbf{m}$ and the orientation vector $\mathbf{o}$ (posterior to anterior) point to opposite directions, i.e., $\boldsymbol{m} \cdot \boldsymbol{o}<0$. Avoiding reactions consisting of a single pair of frames were discarded. The mean frequency of spontaneous avoiding reactions was calculated as the number of avoiding reactions across all trajectories, divided by the total duration, yielding $0.18 \mathrm{~Hz}$.

Only reorientation events involved in planar avoiding reactions were selected, based on measurements of the eccentricity of the ellipse that best fits the shape of paramecia. Whenever this eccentricity went below 0.8 , the event was discarded. The total reorientation angle was obtained by summing all successive instantaneous reorientation angles during the entire avoiding reaction.

In Fig. 6G, the 2D probability density of reorientation angle and backward duration was calculated with Gaussian kernel density estimation.

\section{Deciliation}

Deciliated cells were obtained by adding $96 \%$ ethanol to a tube containing the previously washed and adapted cells in the extracellular solution up to a final concentration of $5 \%(v / v)$ (Ogura, 1981). Then the tube was shaken for $2 \mathrm{~min}$ and left to rest for $1 \mathrm{~min}$. Deciliated cells were collected from the lower half of the tube since they no longer accumulate at the top of the solution. Cilia start to grow back after approximately $30 \mathrm{~min}$. Thus, as described in (Ogura, 1981), in some experiments we blocked cilia regrowth by adding $10 \mathrm{mM}$ of colchicine to the extracellular solution.

\section{Electrophysiology}


The extracellular solution used in all experiments contains $1 \mathrm{mM} \mathrm{CaCl} 2,4 \mathrm{mM} \mathrm{KCl}$ and $1 \mathrm{mM}$ Tris- $\mathrm{HCl}$ buffer with $\mathrm{pH} 7.2$, except for Fig. 2B, where there was no $\mathrm{KCl}$ (blue curve). Microelectrodes of $\sim 50 \mathrm{M} \Omega$ resistance were pulled using a micropipette puller (P-1000, Sutter Instrument) from standard wall borosilicate capillary glass with filament (o.d. $1 \mathrm{~mm}$, i.d. $0.5 \mathrm{~mm}$, Harvard Apparatus). They were filled with a $1 \mathrm{M} \mathrm{KCl}$ solution using a MicroFil non-metallic syringe needle (MF 34G-5, World Precision Instruments); a few recordings were done with $3 \mathrm{M} \mathrm{KCl}$ (no particular change was noticed).

We used an upright microscope (LNScope, Luigs \& Newmann) with two objectives, a $20 \times$ air objective (SLMPLN Plan Achromat, Olympus) to locate cells, and a 40× water immersion objective (LUMPLFLN, Olympus) with DIC contrast enhancement for electrophysiology and imaging.

Paramecia were immobilized using the device described in (Kulkarni et al., 2020). Briefly, paramecia are immobilized against a transparent filter (Whatman Cyclopore polycarbonate membranes; diameter $25 \mathrm{~mm}$, pore diameter $12 \mu \mathrm{m}$ ) thanks to a peristaltic pump (Gilson Minipulse 3) that circulates the fluid from below the filter to above the device. Two microelectrodes are then lowered into the cell, and the pump is stopped. The cell is then held in place by the electrodes.

Electrophysiology recordings were performed using an amplifier with capacitance neutralization (Axoclamp 2B and Axoclamp 900 A, Molecular Device) and an analog-digital acquisition board operating at a sampling frequency of $40 \mathrm{kHz}$ (USB-6343, National Instruments). Custom Python programs (https://github.com/romainbrette/clampy) were used to control the acquisition board.

Membrane potential was recorded with the reading electrode while $100 \mathrm{~ms}$ current pulses of various amplitudes were injected through the second electrode, with at least $1 \mathrm{~s}$ between successive trials.

\section{Particle image velocimetry}

To measure the flows induced by cilia beating, the bath was seeded with $1 \mu \mathrm{m}$ silica or polystyrene particles $(\sim 0.2 \mathrm{mM})$ after paramecia were immobilized and the pump was stopped. Because of sedimentation, particle density was typically higher at the beginning of the experiment. Images were recorded at $30 \mathrm{~Hz}$ with a high-sensitivity CCD camera (Lumenera Infinity 3-6UR) over a $1392 \times 1392$ pixels region of interest surrounding the cell ( 8 bits depth, pixel width $0.178 \mu \mathrm{m}$ ). Frames were synchronized with electrophysiology recordings using a digital trigger.

Frames were preprocessed by removing the background (average image) and band-pass filtering (subtraction of two Gaussian filters with standard deviation $1 \mu \mathrm{m}$ and $1.3 \mu \mathrm{m}$ ). Consecutive frames were then analyzed with particle image velocimetry (PIV) using the OpenPIV Python package (https://github.com/OpenPIV/openpiv-python.git), which calculates the velocity field using image cross-correlation. We used $50 \mu \mathrm{m}$ windows with $2 / 3$ overlap.

In each frame, we calculated the mean angle of the velocity vector over the entire field, using circular mean (argument of the mean complex unit vector; occasional missed frames were discarded). We then subtracted the angle of the anteroposterior axis. The position of anterior and posterior ends was measured manually. As the two ends can be visually ambiguous, they were automatically corrected (by swapping) when the flow measured before stimulation was directed towards the anterior end (indicating backward swimming).

In Figure 5A, for each cell we averaged the mean angle over all currents and over the $300 \mathrm{~ms}$ before stimulus (blue) or over the second half of the stimulus (red), for positive currents $(<5 \mathrm{nA})$. 
$=9$ cells with high quality video recordings and clear cell positioning, indicated by an absence of missed frames and a pre-stimulus flow deviating by less than $45^{\circ}$ from the main cell axis. The average was restricted to responses to large pulses ( 1 to $5 \mathrm{nA}$ ), because those were recorded before the small pulses and therefore had a higher density of particles (due to sedimentation).

\section{Electrophysiological modeling}

In this section, we describe the biophysical models. The parameter values are obtained by fitting the models to the data (section Model fitting).

\section{Electrode model}

All recordings were done with two electrodes, an injecting electrode and a reading electrode. Because of the capacitance and resistance of the injecting electrode, the current injected in the cell is a low-pass filtered version of the command current (Brette et al., 2008). To estimate this current, we model the injecting electrode as a simple RC circuit and estimate its parameters $R_{e}$ and $\tau_{e}$ from responses to small pulses, assuming passive cell responses:

$$
C \frac{d V}{d t}=-g_{L}\left(V-V_{0}\right)+I_{e}
$$

$$
\tau_{e} \frac{d V_{2}}{d t}=V-V_{2}+R_{e} I+\Delta V
$$

$$
I_{e}=\frac{V_{2}-V-\Delta V}{R_{e}}
$$

where $V$ is the membrane potential, assumed identical to the potential of the reading electrode, $V_{2}$ is the potential of the injecting electrode, $I_{e}$ is the current injected in the cell, and $\Delta V$ accounts for a difference in tip potentials. The membrane equation (first equation) is a rough linear model of the cell, but only parameters $R_{e}$ and $\tau_{e}$ are used subsequently, to estimate $I_{e}$ from $I$ according to the last two equations ( $\Delta V$ has no impact on $I_{e}$ and therefore can then be discarded). In the 29 ciliated cells analyzed

\section{Ionic currents}

Paramecium electrophysiology is reviewed in (Eckert and Brehm, 1979) and updated in (Brette, 2021; Valentine and Van Houten, 2022). Paramecium in an isopotential cell (Eckert and Naitoh, 1970; Satow and Kung, 1979). Thus, we consider a single membrane equation:

$$
C \frac{d V}{d t}=I_{L}+I_{K i r}+I_{K d}+I_{C a}+I_{K(C a)}+I
$$

where $C$ is membrane capacitance, $I_{L}=g_{L}\left(E_{L}-V\right)$ is the leak current, $\mathrm{I}_{\mathrm{Kd}}$ is the delayed rectifier $\mathrm{K}^{+}$ current responsible for repolarization, $\mathrm{I}_{\mathrm{Ca}}$ is the ciliary voltage-dependent $\mathrm{Ca}^{2+}$ current, $\mathrm{I}_{\mathrm{K}(\mathrm{Ca})}$ is the calcium-activated $\mathrm{K}^{+}$current, $\mathrm{I}_{\mathrm{Kir}}$ is the inward rectifier $\mathrm{K}^{+}$current and $I$ is a stimulating current. We did not include a few other electrophysiologically identified currents that are less relevant for this 
our extracellular solution does not contain these two ion species), and hyperpolarization-activated 1992a, 1992b), which we did not model.

864 The inward rectifier current $\mathrm{I}_{\mathrm{Kir}}$ is a $\mathrm{K}^{+}$current activated by hyperpolarization, most strongly below $\mathrm{E}_{\mathrm{K}}$ 865 (Oertel et al., 1978). It is modeled as follows:

$$
\begin{aligned}
I_{\text {Kir }} & =g_{\text {Kir }} n_{\text {Kir }}^{p}\left(E_{K}-V\right) \\
\tau_{\text {Kir }} \frac{d n_{\text {Kir }}}{d t} & =\frac{1}{1+\exp \left(\frac{V-V_{\text {Kir }}}{k_{\text {Kir }}}\right)}-n_{\text {Kir }}
\end{aligned}
$$

where $p=1$ or 2 ( $p=2$ in the final version). We made this simple modeling choice because this current was only used as a way to infer the reversal potential $E_{K}$. In particular, we did not include inactivation (Preston et al., 1990). We also tested a version of the model where the linear driving force $\left(E_{K}-V\right)$ is replaced by the Goldman-Hodgkin-Katz expression (Hille, 2001), but it made no significant difference in fitting results.

873 For the delayed rectifier current $I_{K d}$, we tested several models of the type:

$$
I_{K d}=g_{K d} n^{p}\left(E_{K}-V\right)
$$

We tested two classes of models. The Hodgkin-Huxley model is:

$$
\tau_{K d} \frac{d n}{d t}=n_{\infty}(V)-n
$$

where

$$
\alpha_{n}(V)=\frac{a_{K d}}{\operatorname{exprel}\left(\frac{V_{K d}-V}{k_{K d}^{a}}\right)}
$$

$$
\beta_{n}(V)=b_{I K} \exp \left(\frac{V_{K d}-V}{k_{K d}^{b}}\right)
$$

$$
n_{\infty}(V)=\frac{\alpha_{n}(V)}{\alpha_{n}(V)+\beta_{n}(V)}
$$

$$
\tau_{K d}(V)=\tau_{K d}^{\min }+\frac{1}{\alpha_{n}(V)+\beta_{n}(V)}
$$

For numerical stability (near $x=0$ ), we use this special function in the code rather than the explicit expression.

The Boltzmann model is:

$$
n_{\infty}(V)=\frac{1}{1+\exp \left(\frac{V_{K d}-V}{k_{K d}}\right)}
$$




$$
\tau_{K d}(V)=a_{K d}+\frac{b_{K d}}{\cosh \left(\frac{V-V_{\tau}^{K d}}{k_{\tau}^{K d}}\right)}
$$

The voltage-gated calcium current $\mathrm{I}_{\mathrm{Ca}}$ is a calcium-inactivated current located in the cilia (Brehm et al., 1980; Eckert and Brehm, 1979). The corresponding channels have been genetically identified; they are similar to the Cav1 mammalian family (L-type), with a putative calmodulin binding site (Lodh et al., 2016). We model it similarly to (Chad et al., 1984; Standen and Stanfield, 1982):

$$
I_{C a}=g_{C a} m^{2} h f_{G H K}(V)
$$

where $m$ is the activation gating variable, $h$ is the inactivation gating variable, and $f_{G H K}(V)$ is the normalized current-voltage relation of the open channel, given by the Goldman-Hodgkin-Katz equation (Hille, 2001). In the Hodgkin-Huxley model, this relation is linear. However, with very different intracellular and extracellular calcium concentrations, a better model is the GoldmanHodgkin-Katz equation. Resting intracellular concentration is about 50-200 nM (Iwadate, 2003; Klauke and Plattner, 1997), and rises to an estimated $20 \mu \mathrm{M}$ during an action potential (Oertel et al., 1977). In contrast, extracellular concentration is $1 \mathrm{mM}$ in our experiments. Thus, we neglect intracellular concentration, which yields:

$$
f_{G H K}(V)=\frac{1}{\operatorname{exprel}(2 F V / R T)}
$$

where $F$ is the Faraday constant, $R$ is the gas constant, and $T=293 \mathrm{~K}$ is temperature $\left(20^{\circ} \mathrm{C}\right)$. Here, extracellular concentration has been lumped into $g_{C a}$, which is now homogeneous to a current, while $f_{G H K}(V)$ is unitless and has no free parameter.

The activation gating variable is governed by:

$$
\tau_{m} \frac{d m}{d t}=\frac{1}{1+\exp \left(\frac{V_{C a}-V}{k_{C a}}\right)}-m
$$

while the inactivation gating variable is a Hill function of intraciliary calcium concentration $\left[\mathrm{Ca}^{2+}\right]$ :

$$
h\left(\left[\mathrm{Ca}^{2+}\right]\right)=\frac{1}{1+\left(\frac{\left[C a^{2+}\right]}{K_{C a}}\right)^{n_{C a}}}
$$

This is similar to the model of (Standen and Stanfield, 1982), except that the number of sites $n_{C a}$ is allowed to be greater than 1 , because we found that this was necessary to fit our data.

A calcium-activated $\mathrm{K}^{+}$current has been identified by comparison with Pawn mutants lacking functional voltage-activated calcium currents (Satow and Kung, 1980). The current is largely reduced by EGTA. Genomic analysis indicates the presence of both BK and SK channels, with SK channels immunochemically identified in the cilia (Valentine et al., 2012; Yano et al., 2013). We model the current as follows, with activation as a Hill function of $\left[\mathrm{Ca}^{2+}\right]$ :

$$
\begin{gathered}
I_{K(C a)}=g_{K(C a)} m\left(\left[\mathrm{Ca}^{2+}\right]\right)\left(E_{K}-V\right) \\
m\left(\left[\mathrm{Ca}^{2+}\right]\right)=\frac{1}{1+\left(\frac{K_{K(C a)}}{\left[C a^{2+}\right]}\right)^{n_{K(C a)}}}
\end{gathered}
$$




\section{Calcium dynamics}

Resting intracellular calcium concentration $\left[\mathrm{Ca}^{2+}\right]_{0}$ has been estimated between 50 and $200 \mathrm{nM}$ (Iwadate, 2003; Klauke and Plattner, 1997). We chose $\left[\mathrm{Ca}^{2+}\right]_{0}=100 \mathrm{nM}$. Calcium enters the cilia when calcium channels open. The concentration increase is spatially uniform along the cilium (Iwadate and Suzaki, 2004). It then decreases by three mechanisms: buffering, pumps, and diffusion. Buffering can occur with a variety of calcium-binding proteins, an important one being centrin, located in the infraciliary lattice, at the base of cilia (Plattner, 2015; Plattner and Klauke, 2001). Plasma membrane calcium pumps (PMCA) have been identified in the basal membrane with low affinity, around 10-7 M (Wright and van Houten, 1990), and also in the cilia (Yano et al., 2015, 2013). Suppressing the ciliary PCMAs by RNA interference prolongs backward swimming, which means that they are involved in the removal of calcium after an action potential. In principle, calcium can also diffuse to the basal cytosol. However, this has not been observed (Husser et al., 2004). This might be because of cilia volume compared to the cell, or because calcium is buffered at the base of cilia. Both phenomena can be modeled by diffusion to the cilium base, with fixed resting concentration at the boundary.

We lump these diverse mechanisms into two simple processes: a linear process, with rate proportional to $\left(\left[\mathrm{Ca}^{2+}\right]-\left[\mathrm{Ca}^{2+}\right]_{0}\right)$, modelling diffusion and low-affinity buffers, and a high-affinity process operating near rest, with rate given by a Hill function of $\left[\mathrm{Ca}^{2+}\right]$, modelling PMCAs or high affinity buffers. This results in the following equation:

$$
\frac{d\left[\mathrm{Ca}^{2+}\right]}{d t}=\frac{I_{C a}}{2 F v_{\text {cilia }}}+\lambda\left(\left[\mathrm{Ca}^{2+}\right]-\left[\mathrm{Ca}^{2+}\right]_{0}\right)-\frac{J}{1+\frac{\left[\mathrm{Ca}^{2+}\right]_{0}}{\left[\mathrm{Ca}^{2+}\right]}}
$$

where $v$ is the volume of cilia and $\mathrm{F}$ is the Faraday constant. It can be seen that the role of the highaffinity process in this model is to counteract the calcium flow at rest, namely $J=I_{\text {rest }} / F v_{\text {cilia }}$, while the low-affinity process independently tunes the rate of calcium removal after an action potential.

There are 3000 - 4000 cilia in P. tetraurelia (Aubusson-Fleury et al., 2015; Iftode et al., 1989; Nagel and Machemer, 2000), with the upper estimates likely including oral cilia. Each cilium is 10-12 $\mu \mathrm{m}$ long (Eckert and Naitoh, 1970; Sedar and Porter, 1955). Each cilium is $270 \mathrm{~nm}$ wide but the fiber bundle is $200 \mathrm{~nm}$ wide (Aubusson-Fleury et al., 2015). This yields a total volume between 950 and $2750 \mu \mathrm{m}^{3}$. We used the previous estimate $v_{\text {cilia }}=1700 \mu \mathrm{m}^{3}$ from (Oertel et al., 1977), which is compatible with these bounds, but the uncertainty is large. In addition, the effective volume might be smaller because of crowding, or larger because of fast buffering. In practice, an error in the estimation of ciliary volume will translate into an equivalent change in all calcium binding constants (as well as $\lambda$ and $)$ ). Binding constants and volume cannot be determined independently, because the (inverse) volume effectively acts as a unit for those constants.

For stability, the numerical implementation of calcium-dependent equations used equivalent versions written as a function of

$$
p_{C a} \equiv \log \frac{\left[C a^{2+}\right]}{\left[\mathrm{Ca}^{2+}\right]_{0}}
$$

where $[\mathrm{Ca}]_{0}=0.1 \mu \mathrm{M}$ is the resting concentration. For example, inactivation is rewritten as:

$$
h\left(\left[\mathrm{Ca}^{2+}\right]\right)=\frac{1}{1+\exp \left(n_{C a}\left(p_{C a}-p_{K_{C a}}\right)\right)}
$$


This equivalent change of variables avoids numerical issues when $\left[\mathrm{Ca}^{2+}\right]$ approaches 0 . The calcium dynamics equation rewrites as follows:

$$
\frac{d p_{C a}}{d t}=\frac{I_{C a}}{2 F\left[C a^{2+}\right]_{0} v_{\text {cilia }}} e^{-p c a}+\alpha\left(e^{-p C a}-1\right)-\frac{J}{1+e^{p C a}}
$$

\section{Electromotor coupling}

961 Cilia reorient when intraciliary calcium concentration reaches about $1 \mu \mathrm{M}$ (Naitoh and Kaneko, 1972). We model the ciliary angle as a Hill function of $\left[\mathrm{Ca}^{2+}\right]$ :

$$
\alpha=\alpha_{0}+\frac{\Delta \alpha}{1+\left(\frac{K_{\text {motor }}}{\left[C a^{2+}\right]}\right)^{n_{\text {motor }}}}
$$

where angles are relative to the anteroposterior direction ( $\alpha=0$ when cilia beat to the rear), and $K_{\text {motor }}$ is the reversal threshold.

Velocity is modeled as an affine transformation of a Hill function with coefficient $n=2$, changing sign at $K_{\text {motor: }}$ :

$$
v=-v_{\max }+\frac{2 v_{\max }}{1+\left(\frac{\left[C a^{2+}\right]}{K_{\text {motor }}}\right)^{2}}
$$

where $v_{\max }=500 \mu \mathrm{m} / \mathrm{s}$ is maximum velocity (both backward and forward), according to our measurements.

The angle $\theta$ of the rotation axis and the spinning speed $\omega$ are modeled as bell functions peaking at $K_{\text {motor: }}$ :

$$
\begin{aligned}
& \theta=\theta_{\min }+2 \frac{\theta_{\max }-\theta_{\min }}{\left(\frac{K_{\text {motor }}}{\left[C a^{2+}\right]}\right)^{2}+\left(\frac{\left[C a^{2+}\right]}{K_{\text {motor }}}\right)^{2}} \\
& \omega=\omega_{\min }+2 \frac{\omega_{\max }-\omega_{\text {min }}}{\left(\frac{K_{\text {motor }}}{\left[C a^{2+}\right]}\right)^{2}+\left(\frac{\left[C a^{2+}\right]}{K_{\text {motor }}}\right)^{2}}
\end{aligned}
$$

We set $\theta_{\min }=13^{\circ}$ based on our measurements, and $\theta_{\max }=90^{\circ}$, to allow for planar rotations as illustrated in Fig. 5D. We set $\omega_{\min }=2 \pi / \mathrm{s}(1 \mathrm{cycle} / \mathrm{s})$ based on our measurements and $\omega_{\min }=8 \pi / \mathrm{s}(2$ cycles/s), based on the doubling found in the spherical model (Fig. 5E). Thus, no extra free parameter is introduced.

\section{Sensory transduction}

\section{Instantaneous transduction}

In Fig. 7, 8A and 8B where a well-delimited object (half-plane or disc) acts as a stimulus, we first calculate the proportion of the cell surface in contact with the object. To this end, cell shape is determined by the formula proposed by (Zhang et al., 2015): 


$$
y(x)=\frac{b}{2}\left(\sqrt{1-4 \frac{x^{2}}{a^{2}}}-\beta \sin \left(\frac{2 \pi x}{a}\right)\right)
$$

where $x$ is the position along the major axis and $y$ the position along the minor axis, $a=120 \mu \mathrm{m}$ is cell length, $b=35 \mu \mathrm{m}$ is cell width and $\beta=0.15$ is an asymmetry factor. We then simply calculate the intersection of cell and object shapes (as pixel images). The stimulus current is then $I=I_{0} p$, where $I_{0}$ is maximum current and $p$ is the fraction of the cell image within the object.

\section{Delayed transduction}

In Fig. 7B and 8A, we simply consider that the transduction current activates and deactivates with a time constant $\tau_{I}$ :

$$
\tau_{I} \frac{d I}{d t}=I_{0} p-I
$$

This simple model corresponds to channels with finite opening and closing rates (namely, opening rate $\alpha=\tau_{I} s$ and closing rate $\beta=\tau_{I}(1-s)$, where $s$ is proportional to the stimulus $I_{0} p$ ) but does not take into account the spatial recruitment of channels.

Adaptation

In Fig. 8B, $\mathrm{C}$ and 9, we consider that there are two pathways with opposite polarity, a fast pathway and 1001 a slower pathway:

$$
\begin{gathered}
\tau_{\text {fast }} d I_{\text {fast }}=s-I_{\text {fast }} \\
\tau_{\text {slow }} d I_{\text {slow }}=s-I_{\text {slow }} \\
I=I_{\text {slow }}-I_{\text {fast }}
\end{gathered}
$$

where $\tau_{\text {fast }}=40 \mathrm{~ms}$ and $\tau_{\text {slow }}=200 \mathrm{~ms}$. Thus, for a constant stimulus $s$, the stationary current is 0 . In Fig. 8B (disc stimulus), the stimulus is $s=I_{0} p$. In Fig. 8C and 9, where the environment is spatially continuous, the stimulus is simply the value at the center of the cell.

\section{Model optimization}

Model parameters are estimated with the model fitting toolbox of the Brian simulator (Stimberg et al., 2019; Teska et al., 2020) (https://github.com/brian-team/brian2modelfitting). Briefly, the software performs least square optimization using a combination of global optimization algorithms (we used differential evolution) and gradient descent, where the gradient is calculated symbolically from the model equations. Optimization with multiple objectives is done by adding the errors associated to the different objectives. Compiled code is automatically produced by code generation. Each fitting procedure took up to a few hours, and fitting scripts were run in parallel on different cells, using a 
After model fitting, cells were discarded if passive properties were abnormal, indicating a bad recording ( $C>500 \mathrm{pF}$ or $\mathrm{R}<30 \mathrm{M} \Omega$ or $\mathrm{R}>500 \mathrm{M} \Omega$ ), or if $\mathrm{E}_{\mathrm{K}}>\mathrm{E}_{\mathrm{L}}$ (which is biophysically impossible), indicating a fitting problem.

\section{Electrode fitting}

1023

1024

1025

1026

1027

1028

1029

1030

1031

1032

1033

1034

1035

1036

1037

First, for each cell we estimated electrode resistance $R_{e}$ and time constant $\tau_{e}$ from responses to small $100 \mathrm{~ms}$ pulses, both hyperpolarizing and depolarizing $(|\mathrm{I}|<0.5 \mathrm{nA})$, using equations (1-3). The error criterion was the sum of quadratic errors on both electrode potentials, measured from $100 \mathrm{~ms}$ before to $100 \mathrm{~ms}$ after the pulse. The estimated parameters were then used in subsequent fits.

\section{Hyperpolarized fits}

For Fig. 2, we fitted the models described above with $\mathrm{I}_{\mathrm{L}}$ and $\mathrm{I}_{\mathrm{Kir}}$, (equations (4-5)), with least square minimization of the error on the reading electrode potential, taken from pulse start to $50 \mathrm{~ms}$ after the pulse. The stimuli were $100 \mathrm{~ms}$ pulses with amplitude between -4 and $0 \mathrm{nA}$ in $300 \mathrm{pA}$ increments.

We fitted the model with $p=1$ and with $p=2$ for $I_{K i r}$. Using two gates $\left(\mathrm{n}^{2}\right)$ gave better fits than using one ( $\mathrm{n}=40$; median error 2.3 vs. $2.6 \mathrm{mV} ; \mathrm{p}=8.10^{-5}$, one-tailed Wilcoxon test). We also fitted the $\mathrm{n}^{2}$ model with the ohmic driving force replaced by a Goldman-Hodgkin-Katz model, but it did not yield any significant improvement ( $p=0.27$, two-tailed Wilcoxon test). Selection criteria (see above) were passed by $n=28$ cells. Statistics of fitted parameters are shown in Table 1 .

\begin{tabular}{|l|l|l|l|l|}
\hline & Mean & Median & s.d. & s.e.m. \\
\hline $\mathbf{C}(\mathrm{pF})$ & 288.87 & 278.82 & 75.32 & 18.83 \\
\hline $\mathbf{E}_{\mathbf{K}}(\mathrm{mV})$ & -48.27 & -47.92 & 9.54 & 2.38 \\
\hline $\mathbf{E}_{\mathbf{L}}(\mathrm{mV})$ & -20.32 & -19.03 & 11.32 & 2.83 \\
\hline $\mathbf{V}_{\text {Kir }}(\mathrm{mV})$ & -130.27 & -120.75 & 35.36 & 8.84 \\
\hline $\mathbf{g}_{\mathbf{L}}(\mathrm{nS})$ & 9.41 & 7.11 & 6.93 & 1.73 \\
\hline $\mathbf{g}_{\text {Kir }}(\mathrm{nS})$ & 1375.38 & 873.36 & 1206.00 & 301.50 \\
\hline $\mathbf{k}_{\text {Kir }}(\mathrm{mV})$ & 31.53 & 30.23 & 8.89 & 2.22 \\
\hline $\boldsymbol{\tau}_{\text {Kir }}(\mathrm{ms})$ & 15.44 & 15.10 & 3.69 & 0.92 \\
\hline $\mathbf{1} / \mathbf{R}(\mathrm{nS})$ & 1.21 & 1.14 & 0.72 & 0.18 \\
\hline $\mathbf{V}_{\mathbf{0}}(\mathrm{mV})$ & -24.56 & -22.51 & 10.64 & 2.66 \\
\hline
\end{tabular}

Table 1. Statistics of fitted parameters $(n=28)$ for hyperpolarized responses (model with two gates).

\section{Deciliated fits}

For Fig. 3, we fitted the models described above with $\mathrm{I}_{\mathrm{L}}$ and $I_{K d}$, with least square minimization of the error on the reading electrode potential, taken from pulse start to $50 \mathrm{~ms}$ after the pulse. The stimuli were $100 \mathrm{~ms}$ pulses with amplitude between 0 and $4 \mathrm{nA}$ in $300 \mathrm{pA}$ increments. Parameters $E_{K}$ and $C$ were taken from the previous fit to hyperpolarized responses. To make sure the short onset is well captured, the time interval is split in two windows: the first $30 \mathrm{~ms}$ and the rest of the response, and 
each window is equally weighted (meaning that a data point in the first window contributes more than a data point in the second window).

We tested the Hodgkin-Huxley (HH) type model (equations (6-11)) and the Boltzmann model (equations (6-7) and (12-13)). The two models performed similarly ( $\mathrm{n}=21$; median $1.78 \mathrm{mV}$ vs. 1.69 $\mathrm{mV} ; \mathrm{p}=0.59$, two-tailed Wilcoxon test). The median number of gates was 1.13 in the HH model (1.11.8, $25-75 \%$ interval) and 1.7 (1.3-2.7) in the Boltzmann model. In both models, the minimal time constant was very small (median 0.1 vs. $0.6 \mathrm{~ms}$ ). Therefore, we chose a model with 2 gates $\left(n^{2}\right)$ and a nearly null minimal time constant $\left(100 \mu\right.$ s for numerical reasons). A Boltzmann $n^{2}$ model gave similar results to a HH $n^{2}$ model $(p=0.96$, two-tailed Wilcoxon test) and had fewer parameters, while performing similarly to the unconstrained model ( $\mathrm{p}=0.79$, two-tailed Wilcoxon test). Therefore, we chose the Boltzmann $n^{2}$ model. Selection criteria (see above) were passed by $n=16$ cells. Statistics of fitted parameters are shown in Table 2.

Fitting results motivated us to further simplify the model by enforcing $V_{K d}=V_{\tau}$ and $k_{\tau}=2 k_{K d}$. This corresponds to a simple biophysical model where opening and closing rates are of the form $e^{ \pm V / k}$. This simplification slightly increases the fit error (1.82 vs. $1.8 \mathrm{mV} ; \mathrm{p}=0.009$, two-tailed Wilcoxon test), but has the advantage of reducing the parameter set to a single kinetic parameter, the maximum time constant (median $3.2 \mathrm{~ms}$ ).

\begin{tabular}{|l|l|l|l|l|}
\hline & Mean & Median & s.d. & s.e.m. \\
\hline $\mathbf{C}(\mathrm{pF})$ & 158.59 & 145.61 & 48.76 & 12.19 \\
\hline $\mathbf{E}_{\mathbf{K}}(\mathrm{mV})$ & -53.83 & -56.38 & 12.35 & 3.09 \\
\hline $\mathbf{E}_{\mathbf{L}}(\mathrm{mV})$ & -19.68 & -18.33 & 6.48 & 1.62 \\
\hline $\mathbf{V}_{\mathbf{K d}}(\mathrm{mV})$ & 29.77 & 21.31 & 22.86 & 5.71 \\
\hline $\boldsymbol{V}_{\boldsymbol{\tau}_{\boldsymbol{K} d}}(\mathrm{mV})$ & 26.32 & 23.09 & 48.37 & 12.09 \\
\hline $\mathbf{b}_{\mathbf{K d}}(\mathrm{ms})$ & 9.21 & 4.40 & 12.69 & 3.17 \\
\hline $\mathbf{g}_{\mathbf{L}}(\mathrm{nS})$ & 10.62 & 9.72 & 5.73 & 1.43 \\
\hline $\mathbf{g}_{\mathbf{K d}}(\mathrm{nS})$ & 1130.25 & 100.11 & 2656.07 & 664.02 \\
\hline $\mathbf{k}_{\mathbf{K d}}(\mathrm{mV})$ & 8.96 & 6.61 & 6.29 & 1.57 \\
\hline $\boldsymbol{k}_{\boldsymbol{\tau}_{\boldsymbol{K} d}}(\mathrm{mV})$ & 13.54 & 11.74 & 8.08 & 2.02 \\
\hline
\end{tabular}

Table 2. Statistics of fitted parameters ( $n=16)$ for deciliated cells (Boltzmann model with two gates).

\section{Ciliated fits}

1067 For Fig. 4, we fitted the complete model to electrophysiological and ciliary responses to two sets of 100 1068 ms pulses, a set of pulses between 0 and $5 \mathrm{nA}$ in $300 \mathrm{pA}$ increments, and a set of pulses between -100 and $500 \mathrm{pA}$ in $25 \mathrm{pA}$ increments. The complete model consisted of $\mathrm{I}_{\mathrm{L}}$, the simplified $\mathrm{n}^{2}$ Boltzmann model of $I_{K d}$ with $a_{K d}=0.1 \mathrm{~ms}, V_{K_{d}}=V_{\tau}^{K d}$ and $k_{\tau}^{K d}=2 k_{K d}$ (equations (6-7) and (12-13)), $I_{C a}$ (equations (14-16)), $I_{K(C a)}$ (equations (17-18)), calcium dynamics (equation (19)) and electromotor coupling (equation (20)).

1073 To deal with possible shifts in tip potential between the two sets, we aligned all traces to a resting 1074 potential of $-22 \mathrm{mV}$ (the median resting potential), and $\mathrm{E}_{\mathrm{K}}$ was fixed at $-48 \mathrm{mV}$ (the median estimated $1075 \mathrm{E}_{\mathrm{K}}$. 
The optimization error combined an error on the reading electrode potential, an error on the ciliary angle (mean angle of the PIV analysis), and an error on resting calcium concentration. For the voltage error, the response was divided in two equally weighted intervals: from pulse start to pulse end, and from pulse end to $500 \mathrm{~ms}$ after the end (to capture the post-stimulus hyperpolarization). The angle error was defined as the quadratic error on the corresponding unit vectors, which is equivalent to $E_{\alpha}=$ $\cos ^{2}(\alpha-\hat{\alpha})+\sin ^{2}(\alpha-\hat{\alpha})$, and applied on the interval from $100 \mathrm{~ms}$ before the pulse to $500 \mathrm{~ms}$ after it. Finally, we ensured that the resting $\left[\mathrm{Ca}^{2+}\right]$ was $0.1 \mu \mathrm{M}$ by inserting an error on $\left[\mathrm{Ca}^{2+}\right]$ on the interval from $100 \mathrm{~ms}$ before the pulse to the start of the pulse $\left(E_{C a}=\left(\left[\mathrm{Ca}^{2+}\right]-0.1 \mu M\right)^{2}\right)$. This effectively ensures $J=I_{\text {rest }} / F v_{\text {cilia }}$.

Selection criteria (see above) were passed by $n=18$ cells. Statistics of fitted parameters are shown in 1086 Table 3.

\begin{tabular}{|l|l|l|l|l|}
\hline & Mean & Median & s.d. & s.e.m. \\
\hline $\mathbf{C}(\mathrm{pF})$ & 303.06 & 289.10 & 67.81 & 15.98 \\
\hline $\mathbf{E}_{\mathbf{L}}(\mathrm{mV})$ & -23.07 & -23.30 & 1.18 & 0.28 \\
\hline $\mathbf{J}(1 / \mathrm{s})$ & 946.72 & 866.02 & 422.76 & 99.65 \\
\hline $\mathbf{V}_{\mathbf{C a}}(\mathrm{mV})$ & 0.47 & -1.33 & 6.03 & 1.42 \\
\hline $\mathbf{V}_{\mathbf{K d}}(\mathrm{mV})$ & 9.55 & 4.19 & 12.48 & 2.94 \\
\hline $\boldsymbol{\alpha}(1 / \mathrm{s})$ & 19.43 & 7.79 & 32.31 & 7.62 \\
\hline $\mathbf{b}_{\text {Kd }}(\mathrm{ms})$ & 4.77 & 4.50 & 2.58 & 0.61 \\
\hline $\mathbf{g}_{\mathbf{C a}}(\mathbf{n A})$ & 434.81 & 226.63 & 413.79 & 97.53 \\
\hline $\mathbf{g}_{\mathbf{K}(\mathbf{C a})}(\mathbf{n S})$ & 3919.39 & 216.51 & 6499.27 & 1531.89 \\
\hline $\mathbf{g}_{\mathbf{L}}(\mathbf{n S})$ & 10.38 & 9.52 & 4.78 & 1.13 \\
\hline $\mathbf{g}_{\text {Kd }}(\mathbf{n S})$ & 821.80 & 114.76 & 1469.93 & 346.47 \\
\hline $\mathbf{k}_{\mathbf{C a}}(\mathbf{m V})$ & 4.34 & 4.35 & 0.97 & 0.23 \\
\hline $\mathbf{k}_{\text {Kd }}(\mathbf{m V})$ & 5.74 & 4.89 & 2.97 & 0.70 \\
\hline $\mathbf{n}_{\mathbf{C a}}$ & 4.41 & 4.25 & 1.66 & 0.39 \\
\hline $\mathbf{n}_{\mathbf{K}(\mathbf{C a})}$ & 3.53 & 2.94 & 2.35 & 0.55 \\
\hline $\mathbf{n}_{\text {motor }}$ & 7.37 & 7.02 & 5.70 & 1.34 \\
\hline $\mathbf{p K}$ Ca & 3.60 & 3.61 & 0.40 & 0.10 \\
\hline $\mathbf{p K}$ K(Ca) & 6.37 & 6.79 & 1.50 & 0.35 \\
\hline $\mathbf{p K}$ motor & 2.64 & 3.14 & 1.87 & 0.44 \\
\hline $\boldsymbol{\tau}_{\boldsymbol{m}}(\mathrm{ms})$ & 0.91 & 0.94 & 0.38 & 0.09 \\
\hline
\end{tabular}

Table 3. Statistics of fitted parameters $(n=16)$ for depolarized ciliated cells.

\section{Statistics}

1091 Fitting results obtained with different models were compared using the Wilcoxon test. Statistics are given as mean \pm standard deviation. In box plots, the box shows the first and third quartile with the median value inside, and the whiskers are the minimum and maximum values excluding outliers, which are shown as diamonds and defined as those at a distance exceeding 1.5 times the interquartile 


\section{Hydrodynamic model}

In Fig. 5E, we calculated the motion vectors from patterns of ciliary beating on a sphere of $60 \mu \mathrm{m}$ radius. The velocity vector $\boldsymbol{U}$ and the rotation vector $\boldsymbol{\Omega}$ are given by:

$$
\left(\begin{array}{l}
U \\
\Omega
\end{array}\right)=\left(\begin{array}{cc}
M & N \\
N^{T} & O
\end{array}\right)\left(\begin{array}{l}
F \\
\mathbf{L}
\end{array}\right)
$$

where $\mathbf{F}$ is the external force and $\mathbf{L}$ is the external torque (Lauga and Powers, 2009). The matrix is called the mobility matrix. For a sphere of radius $r$, the mobility matrix is diagonal:

$$
\begin{gathered}
\boldsymbol{U}=(6 \pi \eta r)^{-1} \boldsymbol{F} \\
\boldsymbol{\Omega}=\left(8 \pi \eta r^{3}\right)^{-1} \boldsymbol{L}
\end{gathered}
$$

We consider that each patch of membrane is subjected to a force from the fluid, in the direction opposite to the ciliary beating direction, and we calculate the total force and torque for different ciliary beating patterns.

We use spherical coordinates $(\theta, \phi)$ (see Fig. 9), where $\theta=0$ corresponds to the North pole, considered as the anterior end, and $\phi=0$ is the meridian corresponding to the oral groove. Thus, a surface element is:

$$
d S=r^{2} \sin \theta d \theta d \phi
$$

1112 The local force is tangent to the sphere and oriented with an angle $\alpha$, where $\alpha=0$ is the direction of the meridian, pointing South. Cartesian coordinates are chosen so that the $x$ axis is dorso-ventral, the $y$ axis is oriented left to right, and the $z$ axis is posterior to anterior (i.e., South to North pole).

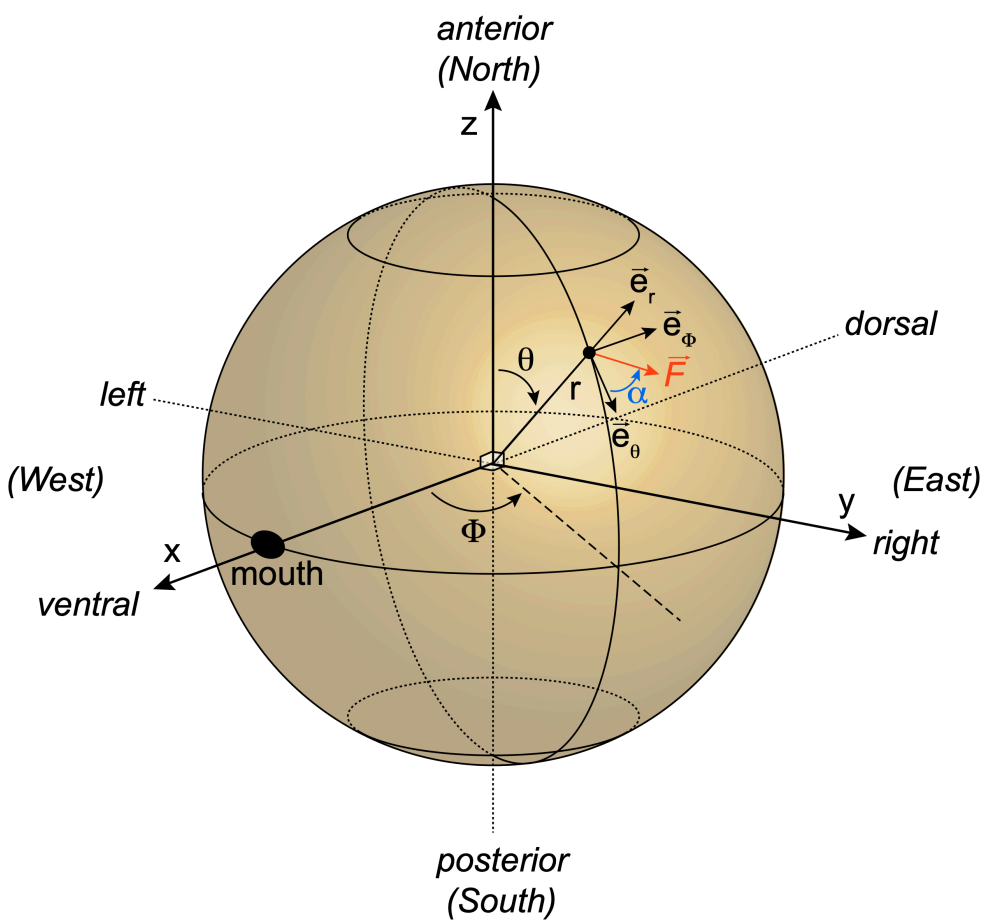

Figure 9. Conventions on the spherical model, with spherical coordinates and a local force $\boldsymbol{F}$ on the surface of the sphere. 
1118 With these conventions, the local force expressed in Cartesian coordinates is:

$$
\boldsymbol{F}=A\left[\begin{array}{c}
\cos \theta \cos \alpha \cos \phi-\sin \alpha \sin \phi \\
\cos \theta \cos \alpha \sin \phi+\sin \alpha \cos \phi \\
-\sin \theta \cos \alpha(\theta, \phi)
\end{array}\right]
$$

where $A$ is the amplitude of the force and $\alpha$ is its angle (to obtain this result, start from the North pole, rotate along the $y$ axis by $\theta$, then rotate along the $z$ axis by $\alpha$ ). Therefore, the total force is:

$$
\boldsymbol{F}_{\text {tot }}=r^{2} \int_{-\pi}^{\pi} \int_{0}^{\pi} \boldsymbol{F}(\theta, \phi) \sin \theta d \theta d \phi
$$

1123 The local torque is $\boldsymbol{\tau}=\boldsymbol{r} \times \boldsymbol{F}$ where $\boldsymbol{r}$ is a radius. In Cartesian coordinates, we obtain:

$$
\begin{gathered}
\boldsymbol{\tau}=r A\left[\begin{array}{c}
-\cos \theta \sin \alpha \cos \phi-\cos \alpha \sin \phi \\
-\cos \theta \sin \alpha \sin \phi+\cos \alpha \cos \phi \\
\sin \theta \sin \alpha
\end{array}\right] \\
\boldsymbol{\tau}_{t o t}=r^{2} \int_{-\pi}^{\pi} \int_{0}^{\pi} \boldsymbol{\tau}(\theta, \phi) \sin \theta d \theta d \phi
\end{gathered}
$$

The reported velocity is $v=\|\boldsymbol{U}\|$, the angle of the rotation axis $\theta_{\text {rotation }}$ is calculated in the xz plane, and the spinning speed is $\omega=\frac{\|\Omega\|}{2 \pi}$ in cycle/s. Local force amplitude is identical in all ciliary beating patterns, and chosen so as to obtain a forward velocity of $500 \mu \mathrm{m} / \mathrm{s}$.

Three different patterns are represented in Fig. 5E. In the forward pattern, the local angle is uniform: $\alpha=-170^{\circ}$, corresponding to a beating direction of $10^{\circ}$, downward to the right. In the backward pattern, the local angle is $\alpha=-10^{\circ}$, corresponding to a beating direction of $170^{\circ}$, upward to the right (obtained by up/down symmetry). In both cases, the local force is axisymmetrical with respect to the anteroposterior axis. It follows that both $\boldsymbol{F}_{\text {tot }}$ and $\boldsymbol{\tau}_{\text {tot }}$ are aligned with the $\mathrm{z}$ axis (anteroposterior). This can be seen in the formulas above by integrating with respect to $\phi$, which yields 0 for the $x$ and $y$ coordinates.

In the turning pattern, the left anterior quarter $\left(\phi \in[-\pi, 0], \theta \in\left[0, \frac{\pi}{2}\right]\right)$ follows the forward pattern $\left(\alpha=-170^{\circ}\right)$, while the right anterior quarter $\left(\phi \in[0, \pi], \theta \in\left[0, \frac{\pi}{2}\right]\right)$ follows the backward pattern $(\alpha=$ $\left.-10^{\circ}\right)$ and the posterior half $(\theta \in[\pi / 2, \pi])$ has local forces pointing to the left $\left(\alpha=-90^{\circ}\right)$, meaning cilia beating to the right. The posterior half generates a rotating pattern around the main axis (by axisymmetry), without translational movement. Each anterior quarter generates forces and torques

$$
\begin{aligned}
& \boldsymbol{F}_{\text {tot }}=\left[\begin{array}{c}
2 \sin \alpha \\
-\cos \alpha \\
-\frac{\pi}{4} \cos \alpha
\end{array}\right] \\
& \boldsymbol{\tau}_{\text {tot }}=r\left[\begin{array}{c}
2 \cos \alpha \\
\sin \alpha \\
\frac{\pi}{4} \sin \alpha
\end{array}\right]
\end{aligned}
$$

1143 With $\alpha_{L}=\pi-\alpha_{R}$, we then find that the $y$ and $z$ components of the total torque vanish. Added to the torque generated by the posterior part, we obtain a torque vector in the $x z$ plane, which is the plane of the oral groove, separating the cell in left and right parts. 


\section{Kinematics}

1148 We consider that the organism is an object moving by rigid motion. The organism is characterized by a position vector $\mathbf{x}$, and an orientation matrix $\mathbf{R}$ defining the rotation of the reference frame (frame of the organism), so that a point $\mathbf{y}$ on the reference frame is mapped to $\mathbf{R y}$ in the observer frame. The reference frame is chosen as in the spherical model above, so that $z>0$ points towards the anterior end while the $x$ axis the dorsoventral axis.

1153 Translational velocity is assumed to be in the posterior-anterior direction only: $v=[0,0, v]$, so that

$$
\dot{x}=R v
$$

Let $\boldsymbol{\omega}$ be the rotation vector in the reference frame. We assume it is tilted from the main axis by an 1156 angle $\theta$ in the $x z$ plane:

$$
\boldsymbol{\omega}=-\omega[\sin (\theta), 0, \cos (\theta)]
$$

1158 Over a time $d t$, the organism rotates by $\boldsymbol{\Omega}(\mathrm{dt})=\mathbf{I}+[\boldsymbol{\omega}] . \mathrm{dt}$, where

$$
[\omega]=\left[\begin{array}{ccc}
0 & -\cos (\theta) & 0 \\
\cos (\theta) & 0 & -\sin (\theta) \\
0 & \sin (\theta) & 0
\end{array}\right]
$$

is the infinitesimal rotation matrix, such that $[\boldsymbol{\omega}] \mathbf{y}=\boldsymbol{\omega} \times \mathbf{y}$. Therefore, the orientation matrix

1161 changes as $\boldsymbol{R}(t+d t)=\boldsymbol{R} \boldsymbol{\Omega}(\mathrm{dt})$, giving:

$$
\dot{\boldsymbol{R}}=\boldsymbol{R}[\boldsymbol{\omega}]
$$

For numerical reasons, we use quaternions instead of matrices (Graf, 2008), implemented with the Python packages quaternion and pyquaternion. Orientation is then represented by a unit quaternion $q$, and kinematic equations translate to:

$$
\dot{\hat{x}}=q \widehat{v} \bar{q}
$$

1167 where $\widehat{\boldsymbol{v}}$ is the pure quaternion with imaginary part $\mathbf{v}$, and

$$
\dot{q}=\frac{1}{2} q \widehat{\boldsymbol{\omega}}
$$

\section{Confinement to a plane}

1171 We constrain the organism to move in a plane. This is done simply by rotating the orientation vector at each time step so that it lies in the plane. Concretely, we calculate the orientation vector in the observer frame:

$$
\widehat{\boldsymbol{p}}=q(\widehat{0,0,1}) \bar{q}
$$

1175 Then we rotate the orientation vector around the axis $\mathbf{u}$ that is orthogonal to both the $z$ axis and $\mathbf{p}$ : $1176 \boldsymbol{u}=(0,0,1) \times \boldsymbol{p}$, by an angle $\theta=\sin ^{-1}\left(\boldsymbol{p}_{z} /\|\boldsymbol{p}\|\right)$. The final orientation quaternion is then $1177 q^{\prime}=Q(\boldsymbol{u}, \theta) q$, where $Q(\boldsymbol{u}, \theta)$ is the rotation of axis $\mathbf{u}$ and angle $\theta$. 


\section{Behavioral scenarios}

In all simulations, the electrophysiological model fitted to the cell shown in Fig. 4 is integrated with Euler method and a time step of $0.1 \mathrm{~ms}$, using Brian 2 (Stimberg et al., 2019). Kinematics were integrated with a time step of $1 \mathrm{~ms}$ for simulations with stimuli with sharp boundaries (Figs. 6, 7, 8A and $8 \mathrm{~B}$ ) and $2 \mathrm{~ms}$ for simulations with spatially continuous stimuli (Figs. 8C and 8D).

\section{Avoiding reaction}

In Fig. 6D, models are simulated in a plane with 2 ms pulse currents of amplitude $0.3,0.5$ and $5 \mathrm{nA}$, at 1 second intervals. In Fig. 6F, the stimulus is a 2 ms current pulse of 0.01 to $10 \mathrm{nA}$ amplitude. In Fig. $6 \mathrm{G}$, the stimulus is a $100 \mathrm{pA}$ pulse of duration 0 to $100 \mathrm{~ms}$. The reorientation angle is calculated as the change in angle before and after the stimulus, averaged over 1 second (the spinning period).

\section{Interaction with a stimulus}

1192 In Fig. 7 and all subsequent figures, trajectories are constrained to a plane. In Fig. 7A and B, the stimulus is a half-plane. It produces an instantaneous depolarizing current, proportional to the fraction of the cell shape inside the stimulus (see Sensory transduction), with maximum 5 nA. In Fig. 7C and D, the stimulus additionally goes through a low-pass filter with time constant $40 \mathrm{~ms}$, representing the activation/deactivation rate of the receptors.

\section{Repelling and attracting discs}

In Fig. $8 \mathrm{~A}$ and $8 \mathrm{~B}$, the stimulus is a disc of radius $1 \mathrm{~mm}$ within a $4 \mathrm{~mm}$ torus. 100 trajectories are simulated for $20 \mathrm{~s}$, with random initial positions. A noisy current is added to the membrane equation so as to produce spontaneous action potentials at the observed rate of $0.2 \mathrm{~Hz}$. It is modeled as an Ornstein-Uhlenbeck process:

with $\tau_{n}=20 \mathrm{~ms}$ and $\sigma_{n}=9 \mathrm{pA}$. Physiologically, this corresponds to the random opening of $\mathrm{K}^{+}$ channels (Moolenaar et al., 1976). This noise is included in all subsequent simulations.

In Fig. 8A, the stimulus produces a depolarizing current with a $40 \mathrm{~ms}$ time constant, as in Fig. 7C and D. In Fig. 8B, the stimulus produces an adapting hyperpolarizing current (see Sensory transduction; $\tau_{\text {fast }}=40 \mathrm{~ms}$ and $\tau_{\text {slow }}=200 \mathrm{~ms}$ ), with a maximum amplitude of $1 \mathrm{nA}$.

\section{Gradient following}

1211 In Fig. 8C, the environment has the topology of a cylindrical surface, i.e., circular in the small dimension $1212(500 \mu \mathrm{m})$ and linear in the long dimension $(25 \mathrm{~mm})$. The stimulus is a linear gradient of $100 \mathrm{pA} / \mathrm{mm}$, with transduction modeled with adaptation as for the attracting disc (Fig. 8B). 
In Fig. 8D, cells produce $\mathrm{CO}_{2}$ by breathing, which then diffuses and acidifies the fluid. This is modelled

1217 by the diffusion equation:

$$
\frac{\partial S}{\partial t}=\alpha \cdot 1_{(x, y) \in \text { cell }}+D \Delta S
$$

where $S$ is the stimulus (homogeneous to a current), $\alpha$ is the production rate and $D$ is the diffusion coefficient of $\mathrm{CO}_{2}$. In water at $25^{\circ} \mathrm{C}, D \approx 0.002 \mathrm{~mm}^{2} / \mathrm{s}$ but we accelerate it by actor 5 to speed up the simulation. The production rate is $\alpha=100 \mathrm{pA} / \mathrm{s}$ in a square pixel of width $20 \mu \mathrm{m}$.

\section{Code and data availability}

1224

1225

1226

1227

1228

\section{9}

1230

1231

1232

1233

1234

1235

1236

1237

1238

1239

1240

1241

1242

1243

1244

1245

1246

1247

Code for electrophysiology experiments, model fitting and figures can be found at https:/github.com/romainbrette/Paramecium-model. Electrophysiology data and analyses, including particle image velocimetry can be found at https://doi.org/10.5281/zenodo.6074166. Behavioral data and code can be found at https://doi.org/10.5281/zenodo.6074480.

\section{Acknowledgments}

We thank Marcel Stimberg for technical assistance and Eric Meyer for providing us with specimens of P. tetraurelia.

This work was supported by Agence Nationale de la Recherche (ANR-20-CE30-0025-01 and ANR-21CE16-0013-02), Programme Investissements d'Avenir IHU FOReSIGHT (Grant ANR-18-IAHU-01), Fondation Pour l'Audition Grant FPA RD-2017-2, CNRS (Défi Mécanobiologie, project PERCEE), and Sorbonne Université (Emergence, project NEUROSWIM).

\section{Competing interests}

The authors have no competing interests to declare.

\section{References}

Aubusson-Fleury A, Cohen J, Lemullois M. 2015. Ciliary heterogeneity within a single cell: the Paramecium model. Methods Cell Biol 127:457-485. doi:10.1016/bs.mcb.2014.12.007

Berg HC. 1975. Bacterial behaviour. Nature 254:389-392. doi:10.1038/254389a0

Berke AP, Turner L, Berg HC, Lauga E. 2008. Hydrodynamic attraction of swimming microorganisms by surfaces. Phys Rev Lett 101:038102. doi:10.1103/PhysRevLett.101.038102

Brehm P, Eckert R, Tillotson D. 1980. Calcium-mediated inactivation of calcium current in Paramecium.J Physiol 306:193-203.

Brette R. 2021. Integrative Neuroscience of Paramecium, a "Swimming Neuron." eNeuro 8:ENEURO.0018- 
1250

1251

1252

1253

1254

1255

1256

1257

1258

1259

1260

1261

1262

1263

1264

1265

1266

1267

1268

1269

1270

1271

1272

1273

1274

1275

1276

1277

1278

1279

1280

1281

1282

1283

1284

Brette R, Piwkowska Z, Monier C, Rudolph-Lilith M, Fournier J, Levy M, Frégnac Y, Bal T, Destexhe A. 2008. High-resolution intracellular recordings using a real-time computational model of the electrode. Neuron 59:379-91. doi:S0896-6273(08)00539-4

Bullington WE. 1930. A further study of spiraling in the ciliate Paramecium, with a note on morphology and taxonomy. J Exp Zool 56:423-449. doi:10.1002/jez.1400560404

Chad J, Eckert R, Ewald D. 1984. Kinetics of calcium-dependent inactivation of calcium current in voltageclamped neurones of Aplysia californica. J Physiol 347:279-300.

Cohen N, Denham JE. 2019. Whole animal modeling: piecing together nematode locomotion. Curr Opin Syst Biol, • Systems biology of model organisms • Systems ecology and evolution 13:150-160. doi:10.1016/j.coisb.2018.12.002

Cohen N, Sanders T. 2014. Nematode locomotion: dissecting the neuronal-environmental loop. Curr Opin Neurobiol, Theoretical and computational neuroscience 25:99-106. doi:10.1016/j.conb.2013.12.003

Drescher K, Goldstein RE, Michel N, Polin M, Tuval I. 2010. Direct Measurement of the Flow Field around Swimming Microorganisms. Phys Rev Lett 105:168101. doi:10.1103/PhysRevLett.105.168101

Dryl S. 1973. Chemotaxis in Ciliate ProtozoaBehaviour of Micro-Organisms. Springer US. pp. 16-30. doi:10.1007/978-1-4684-1962-7_2

Dupre C, Yuste R. 2017. Non-overlapping Neural Networks in Hydra vulgaris. Curr Biol 27:1085-1097. doi:10.1016/j.cub.2017.02.049

Eckert R. 1972. Bioelectric Control of Ciliary Activity. Science 176:473-481. doi:10.1126/science.176.4034.473

Eckert R, Brehm P. 1979. Ionic mechanisms of excitation in Paramecium. Annu Rev Biophys Bioeng 8:353383. doi:10.1146/annurev.bb.08.060179.002033

Eckert R, Chad JE. 1984. Inactivation of Ca channels. Prog Biophys Mol Biol 44:215-267. doi:10.1016/0079-6107(84)90009-9

Eckert R, Naitoh Y. 1970. Passive electrical properties of Paramecium and problems of ciliary coordination. J Gen Physiol 55:467-483.

Gallois B, Candelier R. 2021. FastTrack: An open-source software for tracking varying numbers of deformable objects. PLOS Comput Biol 17:e1008697. doi:10.1371/journal.pcbi.1008697

Graf B. 2008. Quaternions and dynamics. ArXiv08112889 Math-Ph.

Hamel A, Fisch C, Combettes L, Dupuis-Williams P, Baroud CN. 2011. Transitions between three swimming gaits in Paramecium escape. Proc Natl Acad Sci U S A 108:7290-7295. doi:10.1073/pnas.1016687108

Hansma HG. 1974. Biochemical studies on the behavioral mutants of Paramecium aurelia: ion fluxes and ciliary membrane proteins (PhD Thesis). University of California, Santa Barbara.

Hennessey TM, Kung C. 1985. Slow inactivation of the calcium current of Paramecium is dependent on voltage and not internal calcium. J Physiol 365:165-179. doi:10.1113/jphysiol.1985.sp015765

Hennessey TM, Kung C. 1984. An anticalmodulin drug, W-7, inhibits the voltage-dependent calcium current in Paramecium caudatum. J Exp Biol 110:169-181.

$$
41 / 46
$$


Hennessey TM, Rucker WB, McDiarmid CG. 1979. Classical conditioning in paramecia. Anim Learn Behav 7:417-423. doi:10.3758/BF03209695

1289 Hille B. 2001. Ion Channels of Excitable Membranes. Sinauer Associates.

1290 Höfer GF, Hohenthanner K, Baumgartner W, Groschner K, Klugbauer N, Hofmann F, Romanin C. 1997. 1291 Intracellular Ca2+ inactivates L-type Ca2+ channels with a Hill coefficient of approximately 1 and an 1292 inhibition constant of approximately 4 microM by reducing channel's open probability. Biophys J

1293 73:1857-1865. doi:10.1016/S0006-3495(97)78216-X

1294 Hook C, Hildebrand E. 1979. Excitation of paramecium. J Math Biol 8:197-214. doi:10.1007/BF00279722

Houten JV. 1978. Two mechanisms of chemotaxis inParamecium. J Comp Physiol 127:167-174. doi:10.1007/BF01352301

Husser MR, Hardt M, Blanchard M-P, Hentschel J, Klauke N, Plattner H. 2004. One-way calcium spill-over during signal transduction in Paramecium cells: from the cell cortex into cilia, but not in the reverse direction. Cell Calcium 36:349-358. doi:10.1016/j.ceca.2004.02.003

Iftode F, Cohen J, Ruiz F, Rueda AT, Chen-Shan L, Adoutte A, Beisson J. 1989. Development of surface pattern during division in Paramecium. I. Mapping of duplication and reorganization of cortical cytoskeletal structures in the wild type. Development 105:191-211.

Iwadate Y. 2003. Photolysis of caged calcium in cilia induces ciliary reversal in Paramecium caudatum.J Exp Biol 206:1163-1170. doi:10.1242/jeb.00219

Iwadate Y, Katoh K, Asai H, Kikuyama M. 1997. Simultaneous recording of cytosolic Ca2+ levels inDidinium andParamecium during aDidinium attack onParamecium. Protoplasma 200:117-127. doi:10.1007/BF01283288

Iwadate Y, Kikuyama M. 2001. Contribution of Calcium Influx on Trichocyst Discharge in Paramecium caudatum. Zoolog Sci 18:497-504. doi:10.2108/zsj.18.497

Iwadate Y, Suzaki T. 2004. Ciliary reorientation is evoked by a rise in calcium level over the entire cilium. Cell Motil 57:197-206. doi:10.1002/cm.10165

Iwatsuki K, Naitoh Y. 1983. Photobehavior in a Colorless Paramecium. BioScience 33:714-715. doi:10.2307/1309353

Iwatsuki K, Naitoh Y. 1982. Photoresponses in colorlessParamecium. Experientia 38:1453-1454. doi:10.1007/BF01955765

Jana S, Eddins A, Spoon C, Jung S. 2015. Somersault of Paramecium in extremely confined environments. Sci Rep 5. doi:10.1038/srep13148

Jennings. 1906. Behavior of the lower organisms. New York, The Columbia university press, The Macmillan company, agents; [etc., etc.].

Jennings HS. 1906. Behavior of the lower organisms. New York, The Columbia university press, The Macmillan company, agents; [etc., etc.].

Jennings HS. 1904. The behavior of paramecium. Additional features and general relations. J Comp Neurol Psychol 14:441-510. doi:10.1002/cne.920140602

Jennings Herbert S. 1899. Studies on reactions to stimuli in unicellular organisms. ii.- the mechanism of the motor reactions of paramecium. Am J Physiol-Leg Content 2:311-341.

$$
42 / 46
$$


Jennings H. S. 1899. Studies on reactions to stimuli in unicellular organisms. iv.-laws of chemotaxis in paramecium. Am J Physiol-Leg Content 2:355-379. doi:10.1152/ajplegacy.1899.2.4.355 Movements and Reactions of Pieces of Ciliate Infusoria. Biol Bull 3:225-234. doi:10.2307/1535876

Jensen P. 1893. Ueber den Geotropismus niederer Organismen. Arch Für Gesamte Physiol Menschen Tiere 53:428-480. doi:10.1007/BF01706283

Jung I, Powers TR, Valles JM. 2014. Evidence for two extremes of ciliary motor response in a single swimming microorganism. Biophys J 106:106-113. doi:10.1016/j.bpj.2013.11.3703

Klauke N, Plattner H. 1997. Imaging of Ca2+ transients induced in Paramecium cells by a polyamine secretagogue. J Cell Sci 110:975-983.

1337 Knoll G, Haacke-Bell B, Plattner H. 1991. Local trichocyst exocytosis provides an efficient escape 1338 mechanism for Paramecium cells. Eur J Protistol 27:381-385. doi:10.1016/S0932-4739(11)80256-7

1339 Kulkarni A, Elices I, Escoubet N, Pontani L-L, Prevost AM, Brette R. 2020. A simple device to immobilize 1340 protists for electrophysiology and microinjection. J Exp Biol 223. doi:10.1242/jeb.219253

1341 Kung C. 1971. Genic mutants with altered system of excitation in Paramecium aurelia. II. Mutagenesis, screening and genetic analysis of the mutants. Genetics 69:29-45.

Kung C, Saimi Y. 1985. Chapter 3 Ca2+ Channels of Paramecium: A Multidisciplinary Study In: Bronner F, 1344 Kleinzeller A, editors. Current Topics in Membranes and Transport, Genes and Membranes: Transport 1345 Proteins and Receptors. Academic Press. pp. 45-66. doi:10.1016/S0070-2161(08)60149-5

1346 Kunita I, Kuroda S, Ohki K, Nakagaki T. 2014. Attempts to retreat from a dead-ended long capillary by 1347 backward swimming in Paramecium. Front Microbiol 5. doi:10.3389/fmicb.2014.00270

1348 Lauga E, Powers TR. 2009. The hydrodynamics of swimming microorganisms. Rep Prog Phys 72:096601. 1349 doi:10.1088/0034-4885/72/9/096601

1350 Lodh S, Yano J, Valentine MS, Van Houten JL. 2016. Voltage-gated calcium channels of Paramecium cilia. J 1351 Exp Biol 219:3028-3038. doi:10.1242/jeb.141234

1352 Lu Z. 2004. Mechanism of rectification in inward-rectifier K+ channels. Annu Rev Physiol 66:103-129. 1353 doi:10.1146/annurev.physiol.66.032102.150822

1354 Machemer H. 1974. Frequency and directional responses of cilia to membrane potential changes 1355 inParamecium. J Comp Physiol 92:293-316. doi:10.1007/BF00696617

1356 Machemer H. 1972. Ciliary Activity and the Origin of Metachrony in Paramecium: Effects of Increased 1357 Viscosity. J Exp Biol 57:239-259.

1358 Machemer H. 1969. Regulation der Cilienmetachronie bei der "Fluchtreaktion" von Paramecium*.J

1359 Protozool 16:764-771. doi:10.1111/j.1550-7408.1969.tb02340.x

1360 Machemer H, Machemer-Röhnisch S. 1984. Mechanical and electric correlates of mechanoreceptor 1361 activation of the ciliated tail inParamecium. J Comp Physiol A 154:273-278. doi:10.1007/BF00604993

1362 Machemer H, Ogura A. 1979. Ionic conductances of membranes in ciliated and deciliated Paramecium. $J$ 1363 Physiol 296:49-60. 
1364 Machemer-Röhnisch S, Machemer H. 1989. A Ca paradox: Electric and behavioural responses of Paramecium following changes in external ion concentration. Eur J Protistol 25:45-59. doi:10.1016/S0932-4739(89)80077-X

1367 Martinac B, Saimi Y, Kung C. 2008. Ion Channels in Microbes. Physiol Rev 88:1449-1490.

1368 doi:10.1152/physrev.00005.2008

1369 Mendelssohn M. 1895. Ueber den Thermotropismus einzelliger Organismen. Arch Für Gesamte Physiol 1370 Menschen Tiere 60:1-27. doi:10.1007/BF01661667

1371 Moolenaar WH, De Goede J, Verveen AA. 1976. Membrane noise in Paramecium. Nature 260:344-346. doi:10.1038/260344a0

Nagel U, Machemer H. 2000. Physical and physiological components of the graviresponses of wild-type and mutant Paramecium Tetraurelia. J Exp Biol 203:1059-1070.

1375 Naitoh Y, Eckert R. 1972. Electrophysiology of Ciliate ProtozoaExp. in Physiol. and Biochem. pp. 17-31. Naitoh Y, Kaneko H. 1972. Reactivated Triton-Extracted Models of Paramecium: Modification of Ciliary Movement by Calcium Ions. Science 176:523-524. doi:10.1126/science.176.4034.523

1378 Nakaoka Y, Iwatsuki K. 1992. Hyperpolarization-activated inward current associated with the frequency increase in ciliary beating of Paramecium. J Comp Physiol A 170:723-727. doi:10.1007/BF00198983

Nakaoka Y, Machemer H. 1990. Effects of cyclic nucleotides and intracellular Ca on voltage-activated ciliary beating in Paramecium. J Comp Physiol A 166:401-406. doi:10.1007/BF00204813

Noguchi M, Nakamura Y, Okamoto K-I. 1991. Control of ciliary orientation in ciliated sheets from Paramecium-differential distribution of sensitivity to cyclic nucleotides. Cell Motil 20:38-46. doi:https://doi.org/10.1002/cm.970200105 phosphorylation of axonemal proteins in Paramecium caudatum. Cell Motil 45:263-271. doi:https://doi.org/10.1002/(SICI)1097-0169(200004)45:4<263::AID-CM2>3.0.C0;2-J

Oami K. 1996. Distribution of chemoreceptors to quinine on the cell surface of Paramecium caudatum. $J$ Comp Physiol A 179:345-352. doi:10.1007/BF00194988 paramecium. J Membr Biol 43:169-185. doi:10.1007/BF01933477

Oertel D, Schein SJ, Kung C. 1977. Separation of membrane currents using a Paramecium mutant. Nature 268:120-124. doi:10.1038/268120a0

Ogura A. 1981. Deciliation and Reciliation in Paramecium after Treatment with Ethanol. Cell Struct Funct 6:43-50. doi: $10.1247 /$ csf.6.43

1396 Ogura A, Machemer H. 1980. Distribution of mechanoreceptor channels in theParamecium surface 1397 membrane. J Comp Physiol 135:233-242. doi:10.1007/BF00657251

1398 Ohmura T, Nishigami Y, Taniguchi A, Nonaka S, Manabe J, Ishikawa T, Ichikawa M. 2018. Simple mechanosense and response of cilia motion reveal the intrinsic habits of ciliates. Proc Natl Acad Sci 115:3231-3236. doi:10.1073/pnas.1718294115

1401 Oka T, Nakaoka Y, Oosawa F. 1986. Changes in Membrane Potential during Adaptation to External 1402 Potassium Ions in Paramecium Caudatum. J Exp Biol 126:111-117.

$$
44 / 46
$$


Pallasdies F, Goedeke S, Braun W, Memmesheimer R-M. 2019. From single neurons to behavior in the jellyfish Aurelia aurita. eLife 8:e50084. doi:10.7554/eLife.50084

Párducz B. 1967. Ciliary Movement and Coordination in Ciliates In: Bourne GH, Danielli JF, editors.

1406 International Review of Cytology. Academic Press. pp. 91-128. doi:10.1016/S0074-7696(08)60812-8

1407 Plattner H. 2015. Molecular aspects of calcium signalling at the crossroads of unikont and bikont 1408 eukaryote evolution--the ciliated protozoan Paramecium in focus. Cell Calcium 57:174-185.

1409 doi:10.1016/j.ceca.2014.12.002

1410 Plattner H, Klauke N. 2001. Calcium in ciliated protozoa: sources, regulation, and calcium-regulated cell functions. Int Rev Cytol 201:115-208. doi:10.1016/s0074-7696(01)01003-8

1412 Plattner H, Verkhratsky A. 2018. The remembrance of the things past: Conserved signalling pathways link 1413 protozoa to mammalian nervous system. Cell Calcium 73:25-39. doi:10.1016/j.ceca.2018.04.001

1414 Preston RR. 1998. Transmembrane Mg2+ Currents and Intracellular Free Mg2+ Concentration in 1415 Paramecium tetraurelia. J Membr Biol 164:11-24. doi:10.1007/s002329900389

1416 Preston RR. 1990. A magnesium current in Paramecium. Science 250:285-288.

1417 doi:10.1126/science.2218533

1418 Preston RR, Saimi Y, Kung C. 1992a. Calcium current activated upon hyperpolarization of Paramecium 1419 tetraurelia.J Gen Physiol 100:233-251. doi:10.1085/jgp.100.2.233

1420 Preston RR, Saimi Y, Kung C. 1992b. Calcium-dependent inactivation of the calcium current activated upon hyperpolarization of Paramecium tetraurelia. J Gen Physiol 100:253-268.

Preston RR, Saimi Y, Kung C. 1990. Evidence for two K+ currents activated upon hyperpolarization ofParamecium tetraurelia. J Membr Biol 115:41-50. doi:10.1007/BF01869104

Roesle E. 1903. Die Reaktion einiger Infusorien auf einzelne Induktionsschläge. Z Für Allg Physiol 139168.

Saimi Y. 1986. Calcium-dependent sodium currents inParamecium: Mutational manipulations and effects of hyper- and depolarization. J Membr Biol 92:227-236. doi:10.1007/BF01869391

Saimi Y, Hinrichsen RD, Forte M, Kung C. 1983. Mutant analysis shows that the Ca2+-induced K+ current shuts off one type of excitation in Paramecium. Proc Natl Acad Sci 80:5112-5116. doi:10.1073/pnas.80.16.5112

1431 Saimi Y, Ling KY. 1990. Calmodulin activation of calcium-dependent sodium channels in excised membrane patches of Paramecium. Science 249:1441-1444. doi:10.1126/science.2169650

1433 Satow Y, Kung C. 1980. Ca-Induced K+-Outward Current in Paramecium Tetraurelia. J Exp Biol 88:293304.

1435 Satow Y, Kung C. 1979. Voltage Sensitive Ca-Channels and the Transient Inward Current in Paramecium 1436 Tetraurelia.J Exp Biol 78:149-161.

1437 Schafer WR. 2018. The Worm Connectome: Back to the Future. Trends Neurosci 41:763-765.

1438 doi:10.1016/j.tins.2018.09.002

1439 Sedar AW, Porter KR. 1955. The fine structure of cortical components of Paramecium

1440 multimicronucleatum. J Biophys Biochem Cytol 1:583-604. doi:10.1083/jcb.1.6.583 
1441 Standen NB, Stanfield PR. 1982. A Binding-Site Model for Calcium Channel Inactivation that Depends on

1442 Calcium Entry. Proc R Soc Lond B Biol Sci 217:101-110. doi:10.1098/rspb.1982.0097

1443 Stimberg M, Brette R, Goodman DF. 2019. Brian 2, an intuitive and efficient neural simulator. eLife

1444 8:e47314. doi:10.7554/eLife.47314

1445 Teska A, Stimberg M, Brette R. 2020. brian2modelfitting. Zenodo. doi:10.5281/zenodo.4601961

1446 Tominaga T, Naitoh Y. 1992. Membrane Potential Responses to Thermal Stimulation and the Control of

1447 Thermoaccumulation in Paramecium Caudatum. J Exp Biol 164:39-53.

1448 Valentine MS, Rajendran A, Yano J, Weeraratne SD, Beisson J, Cohen J, Koll F, Van Houten J. 2012.

1449 ParameciumBBS genes are key to presence of channels in Cilia. Cilia 1:16. doi:10.1186/2046-2530-1-16

1450 Valentine MS, Van Houten J. 2022. Ion Channels of Cilia: Paramecium as a Model. J Eukaryot Microbiol 1451 e12884. doi:10.1111/jeu.12884

1452 Wan KY. 2020. Synchrony and symmetry-breaking in active flagellar coordination. Philos Trans R Soc Lond

1453 B Biol Sci 375:20190393. doi:10.1098/rstb.2019.0393

1454 Wang H, Swore JJ, Sharma S, Szymanski JR, Yuste R, Daniel TL, Regnier M, Bosma M, Fairhall AL. 2020.

1455 From neuron to muscle to movement: a complete biomechanical model of Hydra contractile behaviors.

1456 bioRxiv 2020.12.14.422784. doi:10.1101/2020.12.14.422784

1457 Wright MV, van Houten JL. 1990. Characterization of a putative Ca2+-transporting Ca2+-ATPase in the 1458 pellicles of Paramecium tetraurelia. Biochim Biophys Acta BBA - Biomembr 1029:241-251.

1459 doi:10.1016/0005-2736(90)90160-P

1460 Yano J, Rajendran A, Valentine MS, Saha M, Ballif BA, Van Houten JL. 2013. Proteomic analysis of the cilia

1461 membrane of Paramecium tetraurelia.J Proteomics 78:113-122. doi:10.1016/j.jprot.2012.09.040

1462 Yano J, Valentine MS, Van Houten JL. 2015. Novel Insights into the Development and Function of Cilia 1463 Using the Advantages of the Paramecium Cell and Its Many Cilia. Cells 4:297-314.

1464 doi:10.3390/cells4030297

1465 Zack GW, Rogers WE, Latt SA. 1977. Automatic measurement of sister chromatid exchange frequency. J

1466 Histochem Cytochem 25:741-753. doi:10.1177/25.7.70454

1467 Zhang P, Jana S, Giarra M, Vlachos PP, Jung S. 2015. Paramecia swimming in viscous flow. Eur Phys J Spec

1468 Top 224:3199-3210. doi:10.1140/epjst/e2015-50078-x 